\title{
PETITES VALEURS PROPRES DES FIBRÉS PRINCIPAUX EN TORES
}

\author{
par \\ Pierre Jammes
}

\begin{abstract}
Résumé. - Soit $M^{n}$ un fibré principal en tores $T^{k}$ sur une variété compacte $N$. On étudie le spectre du laplacien de Hodge-de Rham lors d'effondrements de $M$ sur $N$ tels que la courbure sectionnelle et le diamètre de $M$ vérifient $|K(M)| \leq a$ et $\operatorname{diam}(M)<d$. On montre d'une part que pour tout $k$, il existe des effondrements pour lesquels la première valeur propre du laplacien agissant sur les formes différentielles de degré 1 et 2 est de l'ordre $\operatorname{de} \operatorname{inj}(M)^{2 k}$, et d'autre part que la première valeur propre non nulle du laplacien agissant sur les 1-formes est minorée par $c(n, a, d, N) \cdot \operatorname{Vol}(M)^{2}$ et $c \cdot \operatorname{inj}(M)^{2 k}$ quand $M^{n}$ s'effondre sur $N$.
\end{abstract}

Abstract. - Let $M^{n}$ be a compact manifold of dimension $n$ with free $T^{k}$ action. We consider collapsings of $M$ on $N=M / T^{k}$ such that the sectional curvature and diameter of $M$ satisfy $|K(M)| \leq a$ and $\operatorname{diam}(M)<d$, and give examples of collapsings for all $k$ such that the first non-zero eigenvalue of Laplacian acting on 1-forms and 2-forms of $M$ are bounded above by $c(M)$. $\operatorname{inj}(M)^{2 k}$. Moreover we prove that the first non-zero eigenvalue of Laplacian acting on 1-forms of all principal $T^{k}$-bundle $M$ over $N$ is bounded below by $c(n, a, d, N) \cdot \operatorname{Vol}(M)^{2}$ and $c \cdot \operatorname{inj}(M)^{2 k}$ when $M$ collapses on $N$.

\section{Introduction}

Soit $(M, g)$ une variété riemannienne compacte connexe orientable de dimension $n$. On considère l'opérateur $\Delta=\mathrm{d} \delta+\delta \mathrm{d}$ agissant sur l'espace $\Omega^{p}(M)$ des $p$-formes différentielles sur $M$. Le spectre de cet opérateur forme un ensemble discret de nombres positifs ou nuls qu'on notera

$$
0=\lambda_{p, 0}(M, g)<\lambda_{p, 1}(M, g) \leq \lambda_{p, 2}(M, g) \leq \ldots,
$$

Classification mathématique par sujets (2000). — 58C40 (primary), 58J50, 35P15, 53C20 (secondary). 
où la multiplicité de $\lambda_{p, 0}(M, g)$ est le $p$-ième nombre de Betti de $M$, les autres valeurs propres étant répétées s'il y a multiplicité.

On sait qu'à diamètre borné et courbure de Ricci minorée, la première valeur propre non nulle du laplacien agissant sur les fonctions est uniformément minorée (Gro80, [LY80]). B. Colbois et G. Courtois ont montré dans [CC90 qu'il n'en va pas de même pour les formes différentielles de degré quelconque et qu'à diamètre et courbure sectionnelle bornés, on peut avoir des valeurs propres arbitrairement petites. De plus, ils montrent que ce phénomène de petites valeurs propres est lié au fait que la variété s'effondre, c'est-à-dire que son volume - ou de manière équivalente son rayon d'injectivité — tend vers zéro, ce qui conduit à considérer le problème suivant :

Question 1.1. - Peut-on minorer $\lambda_{p, 1}$ en fonction de bornes sur le diamètre, la courbure et le rayon d'injectivité, notamment lorsque la variété s'effondre?

T. Mantuano donne dans Man08 une minoration très générale du spectre du laplacien de Hodge-de Rham, mais sa dépendance par rapport au rayon d'injectivité n'est pas explicite. S. Chanillo et F. Trèves avaient auparavant donné dans [CT97] une minoration qui permettait d'expliciter le rôle du rayon d'injectivité, mais leur résultat est compromis par une erreur décelée par T. Mantuano. Dans [CC00], B. Colbois et G. Courtois étudient le cas particulier des fibrés en cercles s'effondrant sur leur base et montrent - entre autres choses que les petites valeurs propres sont alors de l'ordre du rayon d'injectivité au carré, qui est alors du même ordre que le volume au carré. On peut donc reformuler la question 1.1 ainsi :

Question 1.2. - Peut-on obtenir une minoration de $\lambda_{p, 1}(M, g)$ asymptotiquement de l'ordre de $\operatorname{inj}(M, g)^{2}$ ou $\operatorname{Vol}(M, g)^{2}$ quand la variété s'effondre?

En dehors du cas des fibrés en cercles, cette question est presque totalement ouverte. Pour les situations de limites adiabatiques sur certains feuilletages (c'est-à-dire lorsque la métrique s'écrit sous la forme $g=g_{H} \oplus \varepsilon^{2} g_{V}$ où $g_{V}$ une métrique sur les espaces tangents $V$ aux feuilles, $g_{H}$ une métrique sur une distribution complémentaire à $V$ ), on sait (For95, ALK00) qu'on peut calculer pour tout entier $k$ le nombre de petites valeurs propres de l'ordre de $\varepsilon^{2 k}$ à l'aide d'une suite spectrale, mais ces effondrements ne sont pas nécessairement à courbure bornée. Dans le cas où la feuille est de dimension 1, on peut cependant obtenir des effondrements à courbure bornée produisant des petites valeurs propres (cf. [Jam10]). On peut trouver une présentation plus détaillée des questions 1.1 et 1.2 dans [Jam05] ou [Jam11.

Le but du présent travail est de donner des éléments de réponse à la question 1.2 en étudiant le spectre des fibrés principaux en tores s'effondrant sur leur base. Nous allons montrer deux résultats. Le premier apporte une réponse 
négative à la question 1.2 en ce qui concerne le rayon d'injectivité. En effet on peut construire pour tout $k \in \mathbb{N}^{*}$ des effondrements pour lesquels certaines valeurs propres décroissent au moins aussi vite que $\operatorname{inj}(M, g)^{2 k}$. Pour cela on utilisera le fait que, si la fibre est de dimension supérieure ou égale à 2 , on peut l'effondrer non par homothétie mais en privilégiant une certaine direction qu'on choisira pour ses propriétés diophantiennes. Ces effondrements permettent aussi de construire des contre-exemples à certaines des estimées de [CT97 :

Théorème 1.3. - Pour tout entier $k \geq 1$ et pour toute variété $(N, h)$ telle que $b_{2}(N) \geq k$, il existe un fibré principal $M$ en tore $T^{k}$ sur $N$, une famille de métrique $\left(g_{\varepsilon}\right)_{\varepsilon \in] 0,1]}$ sur $M$, et des réels strictement positifs $C_{i}(k,(N, h))$, $i=1,2,3$ et $\varepsilon_{0}(k,(N, h))$ tels que la courbure et le diamètre de $\left(M, g_{\varepsilon}\right)$ soient uniformément bornés par rapport à $\varepsilon, \operatorname{Vol}\left(M, g_{\varepsilon}\right)=\varepsilon$ pour tout $\varepsilon$, et

$$
\varepsilon^{2} \leq C_{1} \cdot \lambda_{p, 1}\left(M, g_{\varepsilon}\right) \leq C_{2} \cdot \operatorname{inj}^{2 k}\left(M, g_{\varepsilon}\right) \leq C_{3} \cdot \varepsilon^{2}
$$

pour $p=1$ et 2 , et pour tout $\varepsilon<\varepsilon_{0}$.

De plus, si $b_{1}(N)>b_{2}(M)$, on a aussi pour $p=2$ et 3

$$
\varepsilon^{2} \leq C_{1} \cdot \lambda_{p, b_{1}(N)-b_{2}(M)}\left(M, g_{\varepsilon}\right) \leq C_{2} \cdot \operatorname{inj}^{2 k}\left(M, g_{\varepsilon}\right) \leq C_{3} \cdot \varepsilon^{2} .
$$

Remarque 1.4. - Le théorème 1.3 montre qu'on ne peut pas minorer la première valeur propre du laplacien par une puissance de rayon d'injectivité indépendante de la dimension. Plus précisément, comme on peut construire des variétés de dimension 3 dont le $2^{\mathrm{e}}$ nombre de Betti est arbitrairement grand, il existe pour tout $n \geq 4$ des variétés de dimension $n$ vérifiant (1.1) avec $k=n-3$. On ne peut donc pas espérer obtenir de minoration générale par le rayon d'injectivité avec un exposant inférieur à $2 n-6$ si $n \geq 4$.

Remarque 1.5. - Les effondrements du théorème 1.3 fournissent des contreexemples à certains des résultats de [CT97], en particulier les théorèmes 1.1 et 1.2 (voir exemple 5.9).

Remarque 1.6. - On verra dans la section 5 qu'il existe effectivement des fibrés vérifiant la condition $b_{1}(N)>b_{2}(M)$.

En petite dimension, on peut en déduire un résultat pour les formes de tout degré :

Corollaire 1.7. - Pour $n=5$ et 7 , il existe un fibré $M$ en tores $T^{2}$ sur une variété de dimension $n-2$, une suite de métrique $\left(g_{\varepsilon}\right)$ sur $M$ et une constante $C>0$ telles que la suite $\left(g_{\varepsilon}\right)$ effondre le fibré $M$ sur sa base à courbure et diamètre bornés quand $\varepsilon$ tend vers zéro, et que

$$
\lambda_{p, 1}\left(M, g_{\varepsilon}\right) \leq C \cdot \operatorname{inj}^{4}\left(M, g_{\varepsilon}\right)
$$

pour $1 \leq p \leq n-1$. 
Le second théorème donne, dans le cas des 1-formes, des minorations du spectre en fonction du volume et du rayon d'injectivité :

Théorème 1.8. - Soit deux réels a et d strictement positifs, un entier $n \geq 3$ et $(N, h)$ une variété riemannienne de dimension strictement inférieure à $n$. Il existe des constantes $\varepsilon_{0}(n, a, d,(N, h))>0, C(n, a, d,(N, h))>0$ et $C^{\prime}(n, a, d,(N, h))>0$ telles que si $(M, g)$ est une variété riemannienne de dimension $n$ vérifiant $\operatorname{diam}(M, g) \leq d,|K(M, g)| \leq$ a et si $\pi:(M, g) \rightarrow(N, h)$ est une fibration principale de fibre $T^{k}$ qui soit une $\varepsilon$-approximation de Hausdorff avec $\varepsilon<\varepsilon_{0}$, alors

$$
\lambda_{1,1}(M, g) \geq C \cdot \operatorname{Vol}^{2}(M, g) \geq C^{\prime} \cdot \operatorname{inj}^{2 k}(M, g) .
$$

Remarque 1.9. - L'exposant du volume dans la minoration (1.4) est optimal. On peut par exemple considérer le cas de produit riemannien d'un fibré en cercles qui s'effondre par un tore dont la métrique est fixée mais dont le diamètre est suffisamment petit pour que le fibré produit soit proche de la base pour la distance de Gromov-Hausdorff, les résultats de [CC00 assurent que dans ce cas, $\lambda_{1,1}(M)$ ne peut pas décroître plus lentement que $\mathrm{Vol}^{2}(M)$. L'inégalité (1.1) assure par ailleurs que l'exposant du rayon d'injectivité dans (1.4) est lui aussi optimal.

Remarque 1.10. - On sait déjà que si la fibre est de dimension supérieure ou égale à deux, on ne peut pas obtenir pour les fibrés en tores de majoration de $\lambda_{1,1}(M)$ par le volume au carré comme c'est le cas pour les fibrés en cercles. En effet, on peut construire des exemples d'effondrements de fibrés principaux en tores pour lesquels la première valeur propre du laplacien ne tend pas vers zéro (cf. [Jam03], théorème 1.13 et paragraphe 4.2).

Le théorème 1.8 soulève les deux questions suivantes qui restent ouvertes :

Question 1.11. - Peut-on généraliser ces résultats aux $p$-formes différentielles, pour tout $p$ ?

Question 1.12. - La minoration du spectre par le volume au carré de la variété se généralise-t-elle à d'autres familles de variétés?

Pour démontrer les théorèmes 1.3 et 1.8 , nous commencerons par trois sections préliminaires sur la topologie, la géométrie et le spectre des fibrés principaux en tores.

Dans la section 2, nous définirons un invariant topologique généralisant la classe d'Euler des fibrés en cercles et qui pourra être utilisé pour contrôler le spectre.

La section 3 sera consacrée à l'étude de la géométrie des fibrés principaux en tores. Le résultat principal est que pour démontrer le théorème 1.8 on peut se ramener à une situation géométrique simple. En particulier, on montrera 
qu'une métrique de courbure et diamètre bornés sur le fibré est proche d'une métrique invariante pour laquelle les fibres sont totalement géodésiques (théorème 3.1).

Dans la section 4, nous étudierons comment, dans le cas d'un fibré principal en tore $T^{k}$ muni d'une métrique invariante, on peut se ramener à l'étude des petites valeurs propres du laplacien à celle du spectre du laplacien resteint aux formes différentielles invariantes par l'action de $T^{k}$ (théorème 4.1). Dans le cas des fibrés en cercles, ce théorème améliore sensiblement un résultat semblable obtenu par B. Colbois et G. Courtois ( $\mathbf{C C 0 0}$ ) qui nécessitait des hypothèses de courbure et diamètre bornés.

Les deux dernières sections serons consacrées aux démonstrations des théorèmes 1.3 et 1.8 . Dans la section 5 , nous construirons les exemples d'effondrements annoncés dans le théorème 1.3

Enfin, dans la section 6. nous démontrerons l'inégalité (1.4) du théorème 1.8 en utilisant les résultats des sections précédentes.

Ces travaux ont été en grande partie réalisés à l'Institut de mathématiques de Neuchâtel et au Laboratoire de mathématiques d'Avignon, qu'ils en soient remerciés. Ma gratitude va aussi à Amandine Lacuès pour sa relecture attentive du texte, ainsi qu'au rapporteur anonyme dont les remarques ont permis d'améliorer le texte de de simplifier certaines démonstrations.

\section{Topologie des fibrés principaux en tores}

Nous allons dans cette partie nous attacher à décrire la topologie des fibrés principaux en tores, et en particulier à construire un invariant différentiel qui permettra, comme la classe d'Euler dans la cas des fibrés en cercles, d'étudier le comportement du spectre du laplacien lors d'un effondrement.

Soit $M$ un fibré principal en tores $T^{k}$ sur une base $N$. Le fibré $M$ peut se décomposer en la somme de Whitney de $k$ fibrés en cercles, de classes d'Euler respectives $e_{1}, \ldots, e_{k}$, mais le $k$-uplet $\left(e_{1}, \ldots, e_{k}\right) \in H^{2}(N)^{k}$ dépend du choix de cette décomposition.

Dans le cas d'un fibré en cercles de classe d'Euler $[e]$, on a la propriété suivante (BT82, p.72) : si $\omega$ est une 1-forme de connexion du fibré, alors $d \omega$ est une 2-forme horizontale qui dépend du choix de la connexion sur le fibré, mais qui est, au signe près, le relevé d'un élément de $[e]$. Dans le cas d'un fibré en tores, on va construire un invariant qui généralise cette propriété.

Dans le cas d'un fibré principal quelconque, une forme de connexion $\gamma$ est une 1-forme verticale invariante à valeur dans l'algèbre de Lie $\mathcal{G}$ de la fibre (voir par exemple [Ram05], chapitre 5). Si on se donne un élément $\omega$ de $\mathcal{G}^{*}$ et qu'on applique $\omega$ à l'image de la forme de connexion, on obtient une 1-forme verticale $\omega \circ \gamma$ à valeur réelle. Si Ker $\omega$ est tangent à un sous-tore $T^{k-1} \subset T^{k}$, alors $\omega \circ \gamma$ est le relevé d'une forme de connexion sur $M / T^{k-1}$, et donc $\mathrm{d}(\omega \circ \gamma)$ 
est le relevé de la classe d'Euler $e \in H^{2}(N)$ de ce fibré. Par linéarité, on peut étendre cette construction en une application $\omega \rightarrow e(\omega)$.

Définition 2.1. - On appelle classe d'Euler du fibré principal $T^{k} \hookrightarrow M \stackrel{\pi}{\rightarrow}$ $N$ l'application e $: \mathcal{G}^{*} \rightarrow H^{2}(N, \mathbb{R})$ déterminée par $\mathrm{d}(\omega \circ \gamma)=\pi^{*}(e(\omega))$ où $\gamma$ est une forme de connexion du fibré et $\mathcal{G}$ l'algèbre de Lie de $T^{k}$.

Remarque 2.1. - Si $k=1$ et si $\omega$ est la forme volume du cercle de longueur 1 , alors $e(\omega)$ est la classe d'Euler du fibré.

On a défini ainsi une classe d'Euler en cohomologie de Rham. On peut aussi la définir en cohomologie de Čech en partant du fait que les $T^{k}$-fibrés principaux ont classifiés par les éléments de $H^{1}\left(N, T^{k}\right)$. Comme la suite exacte courte $1 \rightarrow \mathbb{Z}^{k} \rightarrow \mathbb{R}^{k} \rightarrow T^{k} \rightarrow 1$ induit une suite exacte en cohomologie de Čech

$$
H^{1}\left(N, \mathbb{Z}^{k}\right) \rightarrow H^{1}\left(N, \mathbb{R}^{k}\right) \rightarrow H^{1}\left(N, T^{k}\right) \rightarrow H^{2}\left(N, \mathbb{Z}^{k}\right) \rightarrow,
$$

on peut définir la classe d'Euler comme l'image dans $H^{2}\left(N, \mathbb{Z}^{k}\right)$ de l'élément de $H^{1}\left(N, T^{k}\right)$ caractérisant le fibré. L'équivalence entre les deux définitions s'établit en se ramenant par projections au cas des fibrés en cercles.

Exemple 2.2. - Le théorème 1.13 de [Jam03 montre - entre autres choses - qu'un fibré principal en tores $T^{k}$ non trivial dont la base est un tore $T^{2}$ peut s'écrire comme produit d'une nilvariété de dimension 3 et d'un tore $T^{k-1}$. Comme $H^{2}\left(T^{2}\right)$ est de dimension 1, le noyau de $e$ est de dimension $k-1$, ce qui signifie qu'on peut décomposer le fibré en une somme de Whitney de $k$ fibrés en cercles dont $k-1$ sont triviaux. On retrouve donc le fait que le fibré peut s'écrire comme le produit d'un fibré en cercles sur $T^{2}$ et d'un tore de dimension $k-1$.

\section{Géométrie des fibrés principaux en tores}

3.1. Métriques adaptées. - Nous allons ici montrer qu'on peut, dans le but d'obtenir le théorème 1.8 , se ramener à une situation géométrique plus simple. Cette situation est une généralisation de la notion de métrique adaptée définie dans le cas des fibrés en cercles par B. Colbois et G. Courtois ([CC00]) :

Définition 3.1. - On dit que le couple de métriques $(g, h)$ définies sur $M$ et $N$ respectivement est adapté à la fibration principale $T^{k} \hookrightarrow M^{n} \stackrel{\pi}{\rightarrow} N$ si :

1. La fibration $\pi:(M, g) \rightarrow(N, h)$ est une submersion riemannienne;

2. L'action de $T^{k}$ sur $M$ est isométrique;

3. Les fibres sont totalement géodésiques; 
4. Toute 1-forme verticale $\omega$ induite par un élément de $\mathcal{G}^{*}$ vérifie $\mathrm{d} \omega=$ $\pi^{*}(e(\omega))$, où e est la classe d'Euler du fibré.

On veut montrer qu'une métrique de courbure bornée sur un fibré principal en tores est proche d'une métrique adaptée :

Théorème 3.1. - Soient a et d deux réels strictement positifs, et $T^{k} \hookrightarrow$ $\left(M^{n}, g\right) \stackrel{\pi}{\rightarrow}(N, h)$ un fibré principal en tores. Il existe des constantes $\varepsilon_{0}(n, a,(N, h))>0, \tau(n, a,(N, h))>0, \tau^{\prime}(n, a,(N, h))>0$ et $c(n, a,(N, h))>$ 0 telles que si $|K(N, h)| \leq a,|K(M, g)| \leq a$ et si $\pi$ est une $\varepsilon$-approximation de Hausdorff avec $\varepsilon<\varepsilon_{0}$, alors il existe des métriques $\tilde{g}$ et $\tilde{h}$ sur $M$ et $N$ respectivement et une fibration $\pi^{\prime}:(M, \tilde{g}) \rightarrow(N, \tilde{h})$ telles que

1. Le couple $(\tilde{g}, \tilde{h})$ est adapté à la fibration $\pi^{\prime}$;

2. $\frac{1}{\tau} g \leq \tilde{g} \leq \tau g$ et $\frac{1}{\tau} h \leq \tilde{h} \leq \tau h$;

3. La restriction de $\tilde{g}$ à la fibre est telle que $\operatorname{diam}\left(\pi^{\prime-1}(x)\right) \leq \tau^{\prime} \varepsilon$, pour tout $x \in N$;

4. La courbure sectionnelle de $(M, \tilde{g})$ vérifie $|K(X, Y)| \leq c$, pour toute paire de vecteurs horizontaux orthonormés $(X, Y)$.

On pourra alors appliquer le résultat de J. Dodziuk selon lequel si deux métriques sont proches, alors les spectres du laplacien pour ces deux métriques sont proches aussi :

Théorème 3.2 ([Dod82]). - Soit g et $\tilde{g}$ deux métriques riemanniennes sur une variété compacte $M$ de dimension $n$, et $\tau$ une constante strictement positive. Si les deux métriques vérifient $\frac{1}{\tau} g \leq \tilde{g} \leq \tau g$, alors

$$
\frac{1}{\tau^{3 n-1}} \lambda_{p, k}(M, g) \leq \lambda_{p, k}(M, \tilde{g}) \leq \tau^{3 n-1} \lambda_{p, k}(M, g),
$$

pour tous entiers $k \geq 0$ et $p \in[0, n]$.

Remarque 3.3. - En vertu d'un théorème de Hermann ([Her60], Bes08 p. 249), le fait que les fibres soient totalement géodésiques implique qu'elles sont isométriques entre elles. On va voir dans la démonstration du théorème 3.1 que réciproquement, sur les fibrés considérés, si la métrique est invariante et que les fibres sont isométriques alors elles sont totalement géodésiques.

3.2. Situation de métrique invariante. - Nous allons dans un premier temps montrer que si on suppose qu'on a sur $M$ une métrique invariante, elle est proche d'une métrique qui vérifie les points (1) à (3) de la définition 3.1 . Plus précisément : 
Proposition 3.4. - Soit $T^{k} \hookrightarrow\left(M^{n}, g\right) \stackrel{\pi}{\rightarrow}(N, h)$ un fibré principal en tores muni d'une métrique invariante $g$ tel que $\pi$ soit une submersion riemannienne. Pour tout $a>0$ et $d>0$, il existe des constantes $\tau(n, a, d)>0$ et $c(n, a)>0$ telles que si $|K(N, h)| \leq a, K(M, g) \geq-a$ et $\operatorname{diam}(M, g) \leq d$, alors il existe une métrique invariante $\tilde{g}$ sur $M$ telle que la fibration $\pi:(M, \tilde{g}) \rightarrow(N, h)$ soit une submersion riemannienne à fibres totalement géodésiques et

$$
\frac{1}{\tau} g \leq \tilde{g} \leq \tau g
$$

Remarque 3.5. - On peut noter qu'on utilise non pas une hypothèse de courbure bornée sur $M$ mais seulement que la courbure sectionnelle est minorée.

Pour montrer la proposition 3.4, on utilisera les deux lemmes suivants. Le premier est une application directe de la formule de O'Neill :

Lemme 3.2. - Soit $a>0$ et $T^{k} \hookrightarrow\left(M^{n}, g\right) \stackrel{\pi}{\rightarrow}(N, h)$ un fibré principal en tores muni d'une métrique invariante $g$ tel que $\pi$ soit une submersion riemannienne, $|K(N, h)| \leq a$, et $K_{(M, g)}(X, Y) \geq-a$ pour tout couple $(X, Y)$ de vecteurs horizontaux orthonormés. Alors, pour toute 1-forme différentielle verticale $\omega$, on $a$ :

1. $|\mathrm{d} \omega(X, Y)|_{x}^{2} \leq \frac{8 a}{3}|\omega|_{x}^{2}$, pour tout $x \in M$ et tout couple de vecteurs horizontaux orthonormés $X$ et $Y$;

2. $\|\mathrm{d} \omega\|_{\infty} \leq \frac{4 a n(n-1)}{3}\|\omega\|_{\infty}$.

Démonstration. - Soit $x \in M, y=\pi(x), \tilde{X}$ et $\tilde{Y}$ deux champs de $N$ orthonormés en $y$, et $X$ et $Y$ les relevés de $\tilde{X}$ et $\tilde{Y}$ à $M$. La formule de O'Neill (GHL87] p. 127, Bes08] p. 241) donne

$$
K_{N}(\tilde{X}, \tilde{Y})=K_{M}(X, Y)+\frac{3}{4}\left|[X, Y]^{V}\right|^{2}
$$

où $[X, Y]^{V}$ désigne la composante verticale de $[X, Y]$. D'autre part on a, en utilisant le fait que $\omega$ est verticale,

$$
\begin{aligned}
\mathrm{d} \omega(X, Y) & =X \cdot \omega(Y)-Y \cdot \omega(X)-\omega([X, Y]) \\
& =-\omega([X, Y]) .
\end{aligned}
$$

On en déduit :

$$
\begin{aligned}
|\mathrm{d} \omega(X, Y)|_{x}^{2} & =|\omega([X, Y])|_{x}^{2} \leq|\omega|_{x}^{2}\left|[X, Y]{ }^{V}\right|_{x}^{2} \\
& \leq \frac{4}{3}|\omega|_{x}^{2}\left(K_{y}(\tilde{X}, \tilde{Y})-K_{x}(X, Y)\right)
\end{aligned}
$$


Comme chacun des couples $(\tilde{X}, \tilde{Y})$ et $(X, Y)$ est orthonormé en $x$ et $y$, on a les majorations $\left|K_{y}(\tilde{X}, \tilde{Y})\right| \leq a$ et $K_{x}(X, Y) \geq-a$, et donc

$$
|\mathrm{d} \omega(X, Y)|_{x}^{2} \leq \frac{8 a}{3}|\omega|_{x}^{2}
$$

Et comme l'inégalité précédente est vraie quel que soit le choix de $(\tilde{X}, \tilde{Y})$, il en découle finalement

$$
|\mathrm{d} \omega|_{x}^{2} \leq \frac{\operatorname{4an}(n-1)}{3}|\omega|_{x}^{2} \leq \frac{4 \operatorname{an}(n-1)}{3}\|\omega\|^{2},
$$

ce qui achève la démonstration.

Le second lemme montre que dans le cas d'un fibré en cercles, à courbure bornée, la longueur des fibres varie peu d'une fibre à l'autre.

Lemme 3.8. - Soit $S^{1} \hookrightarrow\left(M^{n}, g\right) \stackrel{\pi}{\rightarrow}(N, h)$ un fibré principal en cercles sur $N$, tel que $g$ soit invariante et $\pi$ soit une submersion riemannienne. Pour tout $a>0$ et $d>0$, il existe $\tau(n, a, d)>0$ tel que si $|K(N, h)| \leq a, K(M, g) \geq-a$ et $\operatorname{diam}(M, g) \leq d$, alors pour tout $x, y \in N$, on a

$$
\frac{1}{\tau} l_{y} \leq l_{x} \leq \tau l_{y}
$$

où $l_{x}$ et $l_{y}$ désignent les longueurs des fibres au dessus de $\pi^{-1}(x)$ et $\pi^{-1}(y)$ respectivement.

Démonstration. - On choisit sur le fibré $M$ une 1-forme verticale $\omega$ dont l'intégrale sur chaque fibre est égale à 1 . Soit $U$ le champ vertical induit par l'action de $S^{1}$ qui vérifie $\omega(U)=1$. La norme $|U|$ de ce champ est constante sur chaque fibre, et s'écrit $|U|=\pi^{*} f$, où $f$ est une fonction sur $N$. De plus, en tout point $x$ de $N$, la norme $f(x)$ de $U$ est égale à la longueur de la fibre au dessus de $x$. On va montrer que $f$ est bornée en fonction de $a$ et $d$. Remarque : $\omega$ n'est pas la forme duale de $U$ pour la métrique. Sa norme ponctuelle sur la fibre $\pi^{-1}(x)$ est $|\omega|=\frac{1}{f}$, et on a $U^{b}=f^{2} \omega$.

Soit $x \in N$, et $\tilde{X}$ un vecteur unitaire tangent à $N$ en $x$. Soit $\tilde{X}_{i}$ une base orthonormée de champs de vecteurs au voisinage de $x$, telle que $\mathrm{D} \tilde{X}_{1} \tilde{X}_{1}=0$ sur ce voisinage, et $\tilde{X}_{1 \mid x}=\tilde{X}$. On relève cette base à $T^{H} M$ en notant $X_{i}=\pi^{*}\left(\tilde{X}_{i}\right)$ et $X=X_{1}$. Ces champs vérifient $\left[U, X_{i}\right]=0$. En effet, ces crochets de Lie sont déterminés par $\left[U, X_{i}\right]=\mathcal{L}_{U} X_{i}$, or les champs $X_{i}$ sont $S^{1}$-invariants. On notera par ailleurs $V$ le champ de norme 1 défini par $V=U /|U|$. On a alors $\left[V, X_{i}\right]=\left(\mathrm{d} f\left(X_{i}\right) / f\right) V$.

On va calculer la courbure sectionnelle $K(X, V)$ en fonction de $f$ et de ses variations. On utilisera pour cela les formules de O'Neill (cf. chapitre 9 de Bes08]) qui donnent en particulier

$$
K(X, V)=\left(\left(\mathrm{D}_{X} T\right)_{V} V, X\right)-\left|T_{V} X\right|^{2}+\left|A_{X} V\right|^{2},
$$


avec les notations suivantes :

- le vecteur $A_{X} V$ est la composante horizontale de $\mathrm{D}_{X} V$;

- le vecteur $T_{V} X$ est la composante verticale de $\mathrm{D}_{V} X$;

- le vecteur $T_{V} V$ est la composante horizontale de $\mathrm{D}_{V} V$ (et mesure donc la courbure extrinsèque de la fibre).

On utilisera abondamment la formule de Koszul, qui caractérise la connexion de Levi-Civita,

$$
\begin{aligned}
2\left\langle\mathrm{D}_{Z_{1}} Z_{2}, Z_{3}\right\rangle= & Z_{1} \cdot\left\langle Z_{2}, Z_{3}\right\rangle+Z_{2} \cdot\left\langle Z_{3}, Z_{1}\right\rangle-Z_{3} \cdot\left\langle Z_{1}, Z_{2}\right\rangle \\
& +\left\langle\left[Z_{1}, Z_{2}\right], Z_{3}\right\rangle-\left\langle\left[Z_{1}, Z_{3}\right], Z_{2}\right\rangle-\left\langle\left[Z_{2}, Z_{3}\right], Z_{1}\right\rangle,
\end{aligned}
$$

ainsi que l'orthogonalité de $\left(X_{1}, \cdots, X_{n}, U\right)$ qui annulera les premiers termes du membre de droite de cette formule. Elle donne directement

$$
\left\langle\mathrm{D}_{V} X, V\right\rangle=\langle[V, X], V\rangle=\frac{\mathrm{d} f(X)}{f} \text { et }\left\langle\mathrm{D}_{X} V, X_{i}\right\rangle=-\frac{1}{2}\left\langle\left[X, X_{i}\right], V\right\rangle^{2} .
$$

On en déduit $\left|T_{U} X\right|^{2}=\left(\frac{\mathrm{d} f(X)}{f}\right)^{2}$ et $\left|A_{X} V\right|^{2}=\frac{1}{4} \sum_{i}\left\langle\left[X, X_{i}\right], V\right\rangle^{2}$.

On calcule le terme restant en partant de la formule de Leibniz :

$$
\left\langle\left(\mathrm{D}_{X} T\right)_{V} V, X\right\rangle=\mathrm{D}_{X}\left\langle T_{V} V, X\right\rangle-\left\langle T_{\mathrm{D}_{X} V} V, X\right\rangle-\left\langle T_{V} \mathrm{D}_{X} V, X\right\rangle .
$$

La formule de Koszul donne alors

$$
\left\langle T_{V} V, X\right\rangle=\left\langle\mathrm{D}_{V} V, X\right\rangle=-\langle[V, X], V\rangle=-\frac{\mathrm{d} f(X)}{f}
$$

et donc

$$
\mathrm{D}_{X}\left\langle T_{V} V, X\right\rangle=\left(\frac{\mathrm{d} f(X)}{f}\right)^{2}-\frac{\operatorname{Hess} f(X, X)}{f} .
$$

On obtient aussi que $\mathrm{D}_{X} V=-\frac{1}{2} \sum_{i}\left\langle\left[X, X_{i}\right], V\right\rangle X_{i}$. Comme $T_{X_{i}} V=0$ ([Bes08], p. 239), on en déduit d'une part que $T_{\mathrm{D}_{X} V} V=0$. Et d'autre part, comme $T_{V} X_{i}$ est vertical on a aussi $\left\langle T_{V} \mathrm{D}_{X} V, X\right\rangle=0$. Il reste finalement :

$$
K(X, V)=-\frac{\operatorname{Hess} f(X, X)}{f}+\frac{1}{4} \sum_{i}\left\langle\left[X, X_{i}\right], V\right\rangle^{2} .
$$

Les derniers termes peuvent être majorés en fonction de la courbure. En effet, on a

$$
\left\langle\left[X, X_{i}\right], V\right\rangle=V^{b}\left(\left[X, X_{i}\right]\right)=f \omega\left(\left[X, X_{i}\right]\right)=-f \mathrm{~d} \omega\left(X, X_{i}\right),
$$

et donc, en vertu du lemme 3.2 .

$$
\left\langle\left[X, X_{i}\right], V\right\rangle^{2} \leq f^{2} \frac{8 a}{3}|\omega|^{2}=\frac{8 a}{3} .
$$


Par hypothèse, la courbure sectionnelle de $M$ est minorée par $-a$. On a donc finalement :

$$
\frac{\operatorname{Hess} f(X, X)}{f} \leq\left(\frac{2 n}{3}+1\right) a .
$$

Soient $x$ et $y$ deux points de $N$, et $\gamma$ une géodésique minimisante joignant ces deux points. Notons $\mu$ la fonction définie par

$$
\mu(t)=\ln f \circ \gamma(t) .
$$

En dérivant $\mu$ par rapport à $t$, on obtient :

$$
\mu^{\prime}(t)=\frac{\mathrm{d} f\left(\gamma^{\prime}(t)\right)}{f \circ \gamma(t)}
$$

et

$$
\begin{aligned}
\mu^{\prime \prime}(t) & =-\left(\frac{\mathrm{d} f\left(\gamma^{\prime}(t)\right)}{f \circ \gamma(t)}\right)^{2}+\frac{\mathrm{d} f\left(\mathrm{D}_{\gamma^{\prime}(t)} \gamma^{\prime}(t)\right)+\operatorname{Dd} f\left(\gamma^{\prime}(t), \gamma^{\prime}(t)\right)}{f \circ \gamma(t)} \\
& =-\mu^{\prime}(t)^{2}+\frac{\operatorname{Hess} f\left(\gamma^{\prime}(t), \gamma^{\prime}(t)\right)}{f \circ \gamma(t)} .
\end{aligned}
$$

On a donc, en vertu de la majoration (3.18) :

$$
\mu^{\prime \prime}(t) \leq \frac{\operatorname{Hess} f\left(\gamma^{\prime}(t), \gamma^{\prime}(t)\right)}{f \circ \gamma(t)} \leq\left(\frac{2 n}{3}+1\right) a .
$$

Supposons que $x$ est un point où $f$, et donc $\mu$, atteint son minimum. On a alors $\mu^{\prime}(0)=0$ et donc, en remarquant que $d$ majore le diamètre de $N$,

$$
\mu^{\prime}(t) \leq\left(\frac{2 n}{3}+1\right) a t
$$

et

$$
\mu(t) \leq \frac{2 n+3}{6} a t^{2} \leq \frac{2 n+3}{6} a d^{2} .
$$

Le rapport $\frac{f(y)}{f(x)}$ est donc majoré par une constante $\tau=\exp \left(\frac{2 n+3}{6} a d^{2}\right)$. Comme on a montré cette majoration en prenant pour $x$ un point où $f$ atteint son minimum, elle sera vraie a fortiori pour un $x$ quelconque.

Remarque : si les fibres sont isométriques, alors le champ $U$ est de norme constante. Il est aisé de vérifier à l'aide de (3.10) que $\mathrm{D}_{U} U$ est alors nul, c'està-dire que les fibres sont totalement géodésiques.

de la proposition 3.4 - Le but est en fait de généraliser le lemme 3.8 aux fibrés en tores pour montrer que $g$ est proche d'une métrique pour laquelle toutes les fibres sont isométriques.

Soit $\bar{U} \in \mathcal{G}$ non nul, où $\mathcal{G}$ est l'algèbre de Lie de $T^{k}$, et $U$ le champ vertical induit $\operatorname{par} \bar{U}$ sur $M$. Soit $x_{0} \in N$. On choisit $\bar{U}$ de sorte que $|U|=1$ au dessus 
de $x_{0}$. De plus, on impose à $\bar{U}$ d'avoir un coefficient directeur rationnel, c'està-dire que $\bar{U}$ est colinéaire à un vecteur de $\mathbb{Z}^{k} \subset \mathcal{G}$. L'action du flot associé à $U$ induit alors une fibration $S^{1} \hookrightarrow M \stackrel{\pi}{\rightarrow}\left(M^{\prime}, g^{\prime}\right)$. On peut contrôler la courbure de ce fibré. En effet, en utilisant la formule de O'Neill, on peut écrire

$$
\begin{aligned}
K_{M^{\prime}}(\tilde{X}, \tilde{Y}) & =K_{M}(X, Y)-\frac{3}{4}\left|[X, Y]^{V}\right|^{2} \\
& =K_{M}(X, Y)-\frac{3}{4} \frac{|\mathrm{d} \omega(X, Y)|^{2}}{|\omega|^{2}},
\end{aligned}
$$

où $\tilde{X}$ et $\tilde{Y}$ sont deux vecteurs orthonormés de $M^{\prime}, X$ et $Y$ leurs relevés respectifs sur $M$, et $\omega$ la 1-forme induite par l'action de $T^{k}$ telle que $\omega(U)=1$. Le lemme 3.2 permet de contrôler le dernier terme en fonction de la courbure de $M$, et donc

$$
K_{M^{\prime}}(\tilde{X}, \tilde{Y}) \geq-a-2 a=-3 a .
$$

Le lemme 3.8 assure alors qu'il existe une constante $\tau(n, a, d)$ telle que

$$
\frac{1}{\tau} \leq|U| \leq \tau
$$

Notons $\tilde{g}$ la métrique invariante sur $M$ obtenue en modifiant $g$ dans la direction verticale de sorte que les fibres soient isométriques à $\pi^{-1}\left(x_{0}\right)$, et en conservant la distribution horizontale et la métrique horizontale associées à $g$. Pour cette nouvelle métrique, la norme de $U$ est uniformément égale à 1. La relation (3.27) peut s'écrire

$$
\frac{1}{\tau^{2}} \tilde{g}(U, U) \leq g(U, U) \leq \tau^{2} \tilde{g}(U, U),
$$

Par continuité, les inégalités (3.28) s'étendent à n'importe quel vecteur vertical. Comme $g$ et $\tilde{g}$ sont identiques sur la direction horizontale, on aura finalement

$$
\frac{1}{\tau^{2}} \tilde{g} \leq g \leq \tau^{2} \tilde{g}
$$

Pour conclure, remarquons que si la métrique sur $M$ est telle que les fibres soient isométriques, la fibration $S^{1} \hookrightarrow M \stackrel{\pi}{\rightarrow}\left(M^{\prime}, g^{\prime}\right)$ induite par le champ $U$ est à fibre totalement géodésique. Par continuité, les fibres du fibré $T^{k} \hookrightarrow M \rightarrow N$ sont elles aussi totalement géodésiques.

3.3. Cas général. - On va maintenant démontrer le théorème 3.1. Pour ce faire, on va s'inspirer d'une démonstration d'un théorème de Lott (Lot02], théorème 2), qui utilise les résultats de CFG92.

Soit $g$ une métrique sur $M$ vérifiant les hypothèses du théorème 3.1. Tout d'abord, en utilisant un résultat de régularisation d'Abresch ([CFG92], théorème 1.12), on construit une métrique $g_{1}$ sur $M$ telle que $\frac{1}{\tau_{1}} g \leq g_{1} \leq \tau_{1} g$, $\left|K\left(M, g_{1}\right)\right| \leq a$ et $\left\|\mathrm{D}^{i} R\right\| \leq A_{i}\left(n, a, \tau_{i}\right)$, où $\tau_{1}>1$ est un réel fixé, et $\mathrm{D}$ et $R$ 
désignent respectivement la dérivée covariante et le tenseur de courbure pour la métrique $g_{1}$.

On applique ensuite le théorème 2.6 de [CFG92], qui assure l'existence de constantes $\varepsilon_{0}(n,(N, h)), \kappa(n, A), \kappa^{\prime}(n, A,(N, h))$ et $\kappa_{i}(n, A,(N, h))$ et d'une fibration $\pi^{\prime}:\left(M, g_{1}\right) \rightarrow(N, h)$ tel que si $\pi$ est une $\varepsilon$-approximation de Hausdorff avec $\varepsilon<\varepsilon_{0}(n,(N, h))$, alors :

- pour tout $x \in N$, le diamètre de $\pi^{\prime-1}(x)$ pour la métrique $g^{\prime}$ est inférieur à $\kappa \varepsilon$;

- la seconde forme fondamentale de la fibre vérifie $\left\|I I_{\pi^{-1}(x)}\right\|_{\infty} \leq \kappa^{\prime}$ pour tout $x \in N$;

- la submersion $\pi^{\prime}$ est $\kappa_{i}$-régulière, c'est-à-dire que $\left\|\mathrm{D}^{i} \pi^{\prime}\right\|_{\infty} \leq \kappa_{i}$, pour tout $i \in \mathbb{N}$.

Enfin, pour une telle fibration $\pi^{\prime}$, les parties 3 et 4 de CFG92 donnent la construction d'une métrique $g_{2}$ sur $M$ qui est $T^{k}$-invariante et telle que $\left|\mathrm{D}^{i}\left(g_{2}-g_{1}\right)\right| \leq c(n, A,(N, h), i)$, pour tout $i \in \mathbb{N}$. Cette dernière égalité assure l'existence d'une constante $\tau_{2}(n, A,(N, h))$ telle que $\frac{1}{\tau_{2}} g_{1} \leq g_{2} \leq \tau_{2} g_{1}$, et permet aussi de contrôler la courbure pour la métrique $g_{2}$.

On peut alors appliquer la proposition 3.4, qui nous donne une métrique $g_{3}$ qui vérifie les points (1) à (3) de la définition 3.1. On utilise le fait que comme $M$ est proche de $N$ pour la distance de Gromov-Hausdorff, son diamètre est contrôlé par $\varepsilon$ et le diamètre de $N$.

Pour obtenir la métrique $\tilde{g}$ du théorème 3.1, il reste à modifier la distribution horizontale de manière à ce que $\tilde{g}$ vérifie le point (4) de la définition 3.1. Remarquons tout d'abord que comme l'application $e: \mathcal{G}^{*} \rightarrow \mathcal{H}^{2}(N)$ est linéaire, il suffit de montrer l'égalité $\mathrm{d} \omega=\pi^{\prime *}(e(\omega))$ pour une base de $\mathcal{G}^{*}$. Soit $\left(\omega_{i}\right)$ une base de $\mathcal{G}^{*}$ orthonormée pour la métrique $g_{3}$. Pour chaque $i, \mathrm{~d} \omega_{i}$ s'écrit

$$
\mathrm{d} \omega_{i}=\pi^{* *}\left(\alpha_{i}+\mathrm{d} \beta_{i}\right)
$$

où $\alpha_{i}$ est une forme harmonique et $\beta_{i}$ une forme cofermée. On définit une nouvelle forme verticale $\omega_{i}^{\prime}=\omega_{i}-\pi^{* *}\left(\beta_{i}\right)$. Cette forme vérifie

$$
\mathrm{d} \omega_{i}^{\prime}=\mathrm{d} \omega_{i}-\pi^{\prime *}\left(\mathrm{~d} \beta_{i}\right)=\pi^{\prime *}\left(\alpha_{i}\right) \in \mathcal{H}^{2}(N) .
$$

L'intersection des noyaux des formes $\omega_{i}^{\prime}$ définit une nouvelle distribution horizontale. On définit $\tilde{g}$ comme étant la métrique sur $M$ telle que $\pi^{\prime}:(M, \tilde{g}) \rightarrow$ $(N, h)$ soit une submersion riemannienne et $\tilde{g}=g_{3}$ sur l'espace vertical. Cette métrique vérifie le point (4) de la définition 3.1 du fait de (3.31).

On doit encore vérifier $\tilde{g}$ est proche de $g_{3}$. Remarquons que

$$
\tilde{g}-g_{3}=\sum_{i}\left(\omega^{\prime 2}-\omega^{2}\right)=\sum_{i}\left(2 \pi^{\prime *}\left(\beta_{i}\right) \otimes \omega_{i}+\pi^{\prime *}\left(\beta_{i}\right)^{2}\right) .
$$

Or, B. Colbois et G. Courtois ont montré dans CC00 (lemme A.32) qu'il existe une constante $\kappa(n, a,(N, h))>0$ telle que les formes $\beta_{i}$ telles qu'on les a 
définies vérifient $\left\|\beta_{i}\right\|_{\infty} \leq \kappa$, ce qui permet de conclure. Remarque : le lemme A.32 de [CC00] utilise le fait que pour la métrique $g_{3}$, la norme de la seconde forme fondamentale est contrôlée et que la submersion $\pi^{\prime}$ est $\kappa_{i}$-régulière. Il n'est donc pas évident qu'on puisse obtenir le théorème 3.1 en supposant que la métrique initiale $g$ est invariante et en se passant des résultats de [CFG92.

Enfin, il reste à montrer que la courbure de $(M, \tilde{g})$ reste bornée dans la direction horizontale. Soit $x \in N, \tilde{X}$ et $\tilde{Y}$ deux vecteurs orthonormés tangents à $N$ en $x, \bar{\omega}$ une 1-forme invariante de $T^{k}, \omega$ la 1-forme induite sur $M$ pour la distribution horizontale associée à $g$ et $\omega^{\prime}$ la 1 -forme induite pour la distribution associée à $\tilde{g}$. Ces deux formes vérifient $\mathrm{d} \omega^{\prime}=\pi^{\prime *}(\alpha)$ et $\mathrm{d} \omega=\pi^{\prime *}(\alpha+\mathrm{d} \beta)$, où $\alpha$ est une 2 -forme harmonique de $N$ et $\beta$ une 1-forme de $N$.

D'après la formule de O'Neill, il suffit pour contrôler la courbure sectionnelle $K_{(M, \tilde{g})}\left(\pi^{\prime *}(X), \pi^{\prime *}(Y)\right)$ de majorer la norme de $\left[\pi^{\prime *}(X), \pi^{* *}(Y)\right]^{V}$. Or, on peut écrire d'une part,

$$
\omega^{\prime}\left(\left[\pi^{\prime *}(X), \pi^{\prime *}(Y)\right]^{V}\right)=\mathrm{d} \omega^{\prime}\left(\pi^{\prime *}(X), \pi^{\prime *}(Y)\right)=\alpha(X, Y) .
$$

D'autre part, on a

$$
\|\alpha\|_{\infty} \leq \tau^{\prime}(N, h)\|\alpha\|_{2},
$$

d'après [Li80] (théorème 7), car $\alpha$ est harmonique, et

$$
\|\alpha\|_{2} \leq\|\alpha+\mathrm{d} \beta\|_{2}=\|\mathrm{d} \omega\|_{2} \leq\|\mathrm{d} \omega\|_{\infty},
$$

en utilisant le fait qu'une forme harmonique est le plus petit élément de sa classe de cohomologie pour la norme $L^{2}$. Enfin, le lemme 3.2 permet de contrôler la norme de $\mathrm{d} \omega$ en fonction de $a$ et $\|\omega\|_{\infty}$, et la norme de $\omega$ est contrôlé en fonction de $\left\|\omega^{\prime}\right\|_{\infty}$. Comme la majoration de $\omega^{\prime}\left(\left[\pi^{\prime *}(X), \pi^{\prime *}(Y)\right]^{V}\right)$ obtenue est indépendante du choix de $\bar{\omega}$, on a bien une majoration de $\left|\left[\pi^{\prime *}(X), \pi^{\prime *}(Y)\right]^{V}\right|$ en fonction de $n, a, \varepsilon$ et $(N, h)$.

\section{Formes invariantes et petites valeurs propres}

Nous allons ici démontrer que pour étudier les petites valeurs propres d'un fibré en tores muni d'une métrique invariante, on peut se restreindre aux formes invariantes :

Théorème 4.1. - Soit $k \in \mathbb{N}^{*}, T^{k} \hookrightarrow M \stackrel{\pi}{\rightarrow} N$ un fibré en tore $T^{k}, \bar{g}$ une métrique invariante sur $T^{k}$ et $f$ une fonction sur $N$ strictement positive. Supposons que $M$ est muni d'une métrique $T^{k}$-invariante $g$ telle que pour tout $x \in N$, la restriction $\bar{g}_{x}$ de $g$ à la fibre $\pi^{-1}(x)$ vérifie $\bar{g}_{x} \leq f(x) \cdot \bar{g}$.

Soit $\lambda$ une valeur propre du laplacien agissant sur les formes différentielles de $M$. Si $\lambda<\left(\sup _{x \in N} f(x)\right)^{-1} \cdot \lambda_{0,1}\left(T^{k}, \bar{g}\right)$, alors les formes propres associées sont $T^{k}$-invariantes. 
Remarque 4.2. - On peut montrer que cette estimation est optimale : si on considère un fibré trivial muni d'une métrique produit, on voit que les formes propres de $T^{k}$ de valeur propre $\lambda_{0,1}\left(T^{k}, \bar{g}\right)$ induisent sur le fibré des formes propres de même valeur propre qui ne sont pas invariantes.

Remarque 4.3. - La démonstration du théorème met en évidence le fait que si la multiplicité d'une valeur propre est impaire, alors le sous-espace propre associé contient des formes invariantes.

Remarque 4.4. - Le résultat peut s'étendre à toute action isométrique d'un groupe de Lie compact.

Démonstration. - Soit $E_{\lambda}$ un espace propre du laplacien de Hodge-de Rham sur $M$. Comme $M$ est munie d'une action isométrique de $T^{k}$, on peut décomposer $E_{\lambda}$ en somme de représentations irréductibles $T^{k}$. En notant $T^{k}=\mathbb{R}^{k} / \Gamma$, ces représentations sont classifiées par le réseau dual $\Gamma^{*}$ de $\Gamma$. On a donc $E_{\lambda}=\oplus_{\gamma \in \Gamma^{*}} E_{\lambda, \gamma}$, avec

$$
E_{\lambda, \gamma}=\left\{\omega \in E_{\lambda}, \forall \xi \in \mathbb{R}^{k}, \forall t \in \mathbb{R}, \varphi_{\xi, t}^{*} \omega=e^{i 2 \pi t\langle\gamma, \xi\rangle} \omega\right\},
$$

où $\varphi_{\xi, t}$ est le flot du champ de vecteur $X_{\xi}$ induit par l'action infinitésimale de $\xi$. Cet espace s'écrit aussi

$$
E_{\lambda, \gamma}=\left\{\omega \in E_{\lambda}, \forall \xi \in \mathbb{R}^{k}, \mathcal{L}_{X_{\xi}} \omega=i 2 \pi\langle\gamma, \xi\rangle \omega\right\} .
$$

La formule de Cartan nous donne, pour $\omega \in E_{\lambda, \gamma}$,

$$
\left\langle\mathcal{L}_{X_{\xi}} \omega, \omega\right\rangle=\left\langle\mathrm{d}_{X_{\xi}} \omega+\iota_{X_{\xi}} \mathrm{d} \omega, \omega\right\rangle=\left\langle\iota_{X_{\xi}} \omega, \delta \omega\right\rangle+\left\langle\mathrm{d} \omega, X_{\xi}^{b} \wedge \omega\right\rangle .
$$

En utilisant le fait que $\left|X_{\xi}^{b} \wedge \omega\right|^{2}+\left|\iota_{X_{\xi}} \omega\right|^{2}=\left|X_{\xi}\right|^{2}|\omega|^{2}$, l'inégalité de CauchySchwarz donne

$$
\begin{aligned}
\left|\left\langle\iota_{X_{\xi}} \omega, \delta \omega\right\rangle+\left\langle\mathrm{d} \omega, X_{\xi}^{b} \wedge \omega\right\rangle\right| & \leq\left\|X_{\xi}\right\|_{\infty}\|\omega\|_{L^{2}}\left(\|\delta \omega\|_{L^{2}}^{2}+\|\mathrm{d} \omega\|_{L^{2}}^{2}\right)^{1 / 2} \\
& \leq\left\|X_{\xi}\right\|_{\infty} \lambda^{1 / 2}\|\omega\|_{L^{2}}^{2} .
\end{aligned}
$$

En combinant 4.2 et 4.4, on obtient que $2 \pi\langle\gamma, \xi\rangle \leq\left\|X_{\xi}\right\|_{\infty} \lambda^{1 / 2}$.

On peut fixer le réseau $\Gamma$ de sorte que la métrique quotient sur $T^{k}$ soit la métrique $\bar{g}$. Le spectre de $\left(T^{k}, \bar{g}\right)$ (pour les fonctions) est alors $\left\{4 \pi^{2}|\gamma|^{2}, \gamma \in\right.$ $\left.\Gamma^{*}\right\}$ (cf. GHL87). Pour un $\xi$ donné de norme 1 , la norme de $X_{\xi}$ est 1 pour la métrique $\bar{g}$ donc, $\left\|X_{\xi}\right\|_{\infty}^{2} \leq \sup _{x \in N} f(x)$. Si $\gamma \neq 0$ et qu'on choisit $\xi$ de norme 1 tel que $\langle\gamma, \xi\rangle=|\gamma|$, on a alors

$$
\lambda_{0,1}\left(T^{k}, \bar{g}\right) \leq 4 \pi^{2}|\gamma|^{2} \leq \sup _{x \in N} f(x) \lambda .
$$

Si $\lambda<\sup _{x \in N} f(x)^{-1} \cdot \lambda_{0,1}\left(T^{k}, \bar{g}\right)$, alors $\gamma=0$ et $E_{\lambda}$ est constitué de formes invariantes. 
de la remarque 4.3. - Il suffit de remarquer que dans la démonstration du théorème, si la dimension du sous-espace propre $E_{\lambda}$ est impaire la décomposition de cet espace représentations irréductibles contient nécessairement la représentation triviale, et donc $E_{\lambda}$ contient des formes invariantes.

\section{Petites valeurs propres et rayon d'injectivité}

Nous allons maintenant démontrer le théorème 1.3. Le cas $k=1$ découlant de CC00, nous supposerons $k \geq 2$. Soit $N$ une variété telle que $b_{2}(N) \geq k$. On se donne un fibré principal en tores $M$ sur $N$ tel que l'application $e: \mathcal{G}^{*} \rightarrow H^{2}(N)$ définie dans la section 2 soit injective (c'est possible car $b_{2}(N) \geq k$ ).

On se donne sur $M$ et $N$ des métriques $g$ et $h$ telles que le couple $(g, h)$ soit adapté (au sens défini en 3.1) à la fibration $M \rightarrow N$ et que la restriction $\bar{g}$ de la métrique $g$ à la fibre $T^{k}$ soit le quotient de la métrique canonique de $\mathbb{R}^{k}$ à $T^{k}=\mathbb{R}^{k} / \mathbb{Z}^{k}$ (on a vu dans la section 3 que le fait que les fibres soient totalement géodésiques est équivalent au fait qu'elles soient isométriques entre elles). On va construire la famille de métriques $\left(g_{\varepsilon}\right)$ sur $M$ en faisant varier la métrique $g_{\varepsilon}$ le long de la fibre, la connexion et la composante horizontale de la métrique restant identiques à celles de $g$.

Soit $y \in \mathbb{R}^{k-1}$ un $(k-1)$-uplet difficilement approchable, c'est-à-dire tel qu'il existe une constante $c(y)$ telle que pour tout $p \in \mathbb{Z}^{k-1}$ et tout $q \in \mathbb{Z}$, on a

$$
\|p-q y\|^{k-1}|q| \geq c(y),
$$

la norme considérée étant la norme euclidienne canonique. On sait qu'il existe une infinité non dénombrable de tels $(k-1)$-uplet (voir Sch80, p. 22 et p. 41-43). On se donne une base orthonormée $\left(X_{1}, \ldots, X_{k}\right)$ de $\mathbb{R}^{k}$ telle que $X_{1}$ soit colinéaire au vecteur $(1, y)$. Pour tout $\varepsilon$, on se donne la base $\mathcal{B}_{\varepsilon}=$ $\left(\frac{1}{\varepsilon} X_{1}, X_{2}, \ldots, X_{k}\right)$ et on définit la métrique $\bar{g}_{\varepsilon}$ sur $\mathbb{R}^{k}$ en posant que $\mathcal{B}_{\varepsilon}$ est orthonormée pour $\bar{g}_{\varepsilon}$, c'est-à-dire qu'on obtient $\bar{g}_{\varepsilon}$ en contractant les longueurs d'un rapport $\varepsilon$ dans la direction de $X_{1}$. Le passage de $\bar{g}_{\varepsilon}$ au quotient $\mathbb{R}^{k} / \mathbb{Z}^{k}$ détermine une métrique sur $T^{k}$, qui elle-même induit la métrique $\left(g_{\varepsilon}\right)$ sur $M$. On a en particulier $g_{1}=g$. Les résultats classiques sur les effondrements (Car84, CG86 théorème 2.1) assurent que la courbure de $\left(M, g_{\varepsilon}\right)$ reste bornée quand $\varepsilon$ tend vers zéro.

On va montrer l'inégalité 1.1 du théorème 1.3 en estimant successivement le volume, le rayon d'injectivité et la première valeur propre de $M$ en fonction de $\varepsilon$.

Fait 5.1. - Pour tout $\varepsilon \in] 0,1]$, on a $\operatorname{Vol}\left(M, g_{\varepsilon}\right)=\operatorname{Vol}(N, h) \cdot \varepsilon$.

Démonstration. - Il suffit de remarquer que $\operatorname{Vol}\left(M, g_{\varepsilon}\right)=\operatorname{Vol}(N, h)$. $\operatorname{Vol}\left(T^{k}, \bar{g}_{\varepsilon}\right)$, et que $\operatorname{Vol}\left(T^{k}, \bar{g}_{\varepsilon}\right)=\operatorname{Det}_{\mathcal{B}_{\varepsilon}} \mathcal{B}_{1}=\varepsilon$. 
Pour obtenir le théorème 1.3 , on aura à reparamétrer $g_{\varepsilon}$ de sorte que $\operatorname{Vol}\left(M, g_{\varepsilon}\right)=\varepsilon$.

Fait 5.2. - Il existe des constantes $\varepsilon_{0}(y, k)>0$ et $C(y, k)>0$ telle que $\operatorname{inj}\left(T^{k}, \bar{g}_{\varepsilon}\right) \geq C \cdot \varepsilon^{\frac{1}{k}}$ pour tout $\varepsilon<\varepsilon_{0}$.

Démonstration. - Calculer le rayon d'injectivité de $\left(T^{k}, \bar{g}_{\varepsilon}\right)$ revient à calculer le minimum des normes dans $\mathbb{R}^{k}$ des points non nuls du réseau $\mathbb{Z}^{k}$ pour la métrique $\bar{g}_{\varepsilon}$. Plus précisément, on peut écrire

$$
2 \operatorname{inj}\left(T^{k}, \bar{g}_{\varepsilon}\right)=\min _{(p, q) \in \mathbb{Z}^{k-1} \times \mathbb{Z} \backslash(0,0)}\|(p, q)\|_{\bar{g}_{\varepsilon}} .
$$

Soit $(p, q) \in \mathbb{Z}^{k-1} \times \mathbb{Z} \backslash(0,0)$. On note $z$ la projection orthogonale de $(p, q)$ sur la droite vectorielle $D$ engendrée par $X_{1}$ (remarque : elle ne dépend pas de $\varepsilon$ ), $\theta$ l'angle entre $D$ et l'hyperplan $\mathbb{R}^{k-1} \times\{0\}$ pour la métrique canonique (on a $\left.\tan \theta=\|y\|^{-1}\right)$ et $\theta^{\prime}$ l'angle entre $D$ et le vecteur $(p, q)-(q \cdot y, q)$ (voir figure 1). De plus, on notera $\|\cdot\|_{\bar{g}_{\varepsilon}}$ la norme pour la métrique $g_{\varepsilon}$ et $\|\cdot\|$ la norme euclidienne canonique. On peut écrire :

$$
\|(p, q)\|_{\bar{g}_{\varepsilon}}^{2}=\|(p, q)-z\|_{\bar{g}_{\varepsilon}}^{2}+\|z\|_{\bar{g}_{\varepsilon}}^{2} .
$$

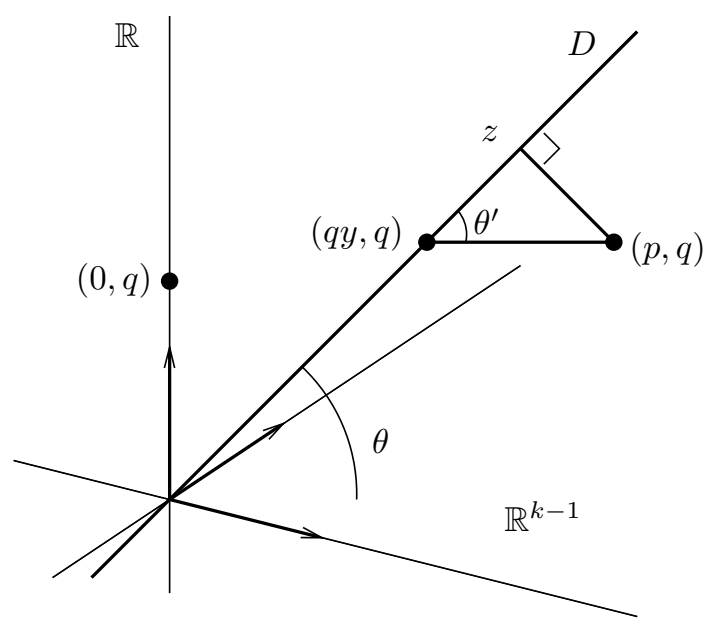

FiguRE 1.

Le vecteur $(p, q)-z$ est orthogonal à $D$, donc la norme $\|(p, q)-z\|_{\bar{g}_{\varepsilon}}$ est indépendante de $\varepsilon$. On a donc, en utilisant la relation (5.1) :

$$
\|(p, q)-z\|_{\bar{g}_{\varepsilon}}=\|(p, q)-z\|=\sin \theta^{\prime}\|p-q y\| \geq \frac{\sin \theta^{\prime} \cdot c(y)^{\frac{1}{k-1}}}{|q|^{\frac{1}{k-1}}} .
$$


Comme $\theta \leq \theta^{\prime} \leq \frac{\pi}{2}$ on en déduit

$$
\|(p, q)-z\|_{\bar{g}_{\varepsilon}}^{2} \geq \frac{a}{|q|^{\frac{2}{k-1}}}
$$

où $a$ est une constante ne dépendant que de $k$ et $y$.

Le vecteur $z$ est parallèle à $D$, donc $\|z\|_{\bar{g}_{\varepsilon}}=\varepsilon\|z\|$. De plus, on a

$$
\|z\| \geq\|(q, q y)\|-\|z-(q, q y)\| \geq \frac{\|q y\|}{\cos \theta}-\|z-(q, q y)\| .
$$

On peut supposer que $\|z-(q, q y)\| \leq \frac{\|q y\|}{2 \cos \theta}$. En effet, dans le cas contraire, on a $\|(p, q)-z\|_{\bar{g}_{\varepsilon}} \geq \sin \theta\|p-q y\| \geq \frac{\sin \theta}{2 \cos \theta}\|q y\| \geq \frac{q}{2}$, ce qui signifie si $q \neq 0$ que $\|(p, q)\|_{\bar{g}_{\varepsilon}}^{2}$ est minoré par $\frac{1}{2}$ et donc ne réalise pas le minimum en 5.2 quand $\varepsilon$ est suffisamment petit (si $q=0$, c'est $\sin \theta\|p-q y\|$ qui est uniformément minoré). On obtient finalement la minoration

$$
\|z\|_{\bar{g}_{\varepsilon}}^{2} \geq \varepsilon^{2} \frac{\|q y\|^{2}}{4 \cos ^{2} \theta}=\varepsilon^{2} b q^{2},
$$

où $b$ est une constante qui ne dépend que de $y$, et donc

$$
\|(p, q)\|_{\bar{g}_{\varepsilon}}^{2} \geq \frac{a}{|q|^{\frac{2}{k-1}}}+\varepsilon^{2} b q^{2} .
$$

La fonction $f: t \rightarrow a t^{-\frac{2}{k-1}}+\varepsilon^{2} b t^{2}$ admet sur $\mathbb{R}_{+}^{*}$ un unique minimum en $t=\left(\frac{a}{\varepsilon^{2} b(k-1)}\right)^{\frac{k-1}{2 k}}$ dont la valeur est $\varepsilon^{2} a^{\frac{k-1}{k}} b^{\frac{1}{k}}\left((k-1)^{\frac{1}{k}}+(k-1)^{\frac{1-k}{k}}\right)$. On peut donc en déduire que

$$
\|(p, q)\|_{\bar{g}_{\varepsilon}} \geq C \varepsilon^{\frac{1}{k}}
$$

où $C$ est une constante ne dépendant que de $y$ et $k$.

Remarque 5.3. - Il découle du second théorème du corps convexe de Minkowski (voir ch. 4 de $\mathbf{S c h 8 0}$, en particulier le lemme 1D) qu'il existe une constante $c>0$ telle que pour toute métrique plate sur $T^{k}$, on a $\operatorname{inj}\left(T^{k}\right)^{k-1} \cdot \operatorname{diam}\left(T^{k}\right) \leq c \cdot \operatorname{Vol}\left(T^{k}\right)$. On peut donc déduire du fait 5.2 que $\operatorname{diam}\left(T^{k}, \bar{g}_{\varepsilon}\right)=O\left(\varepsilon^{1 / k}\right)$.

Remarque 5.4. - Comme la fibre est totalement géodésique, son rayon d'injectivité est égal au rayon d'injectivité de $M$ si $\varepsilon$ est suffisamment petit.

fait 5.5. - Il existe une constante $C^{\prime}(N, k, e)>0$ telle que pour tout $\varepsilon$, on a $\lambda_{p, 1}\left(M, g_{\varepsilon}\right) \leq C^{\prime} \cdot \varepsilon^{2}$ pour $p=1$ et 2 .

Démonstration. - Commençons par le cas $p=1$. Pour estimer la première valeur propre non nulle, on va d'abord calculer quelles sont les 1-formes harmoniques. On sait déjà d'après les résultats de la section précédente que les 
formes harmoniques sont $T^{k}$-invariantes. Soit $\varphi$ une 1-forme différentielle $T^{k}$ invariante de $M$. On peut écrire

$$
\varphi=\pi^{*}(\alpha)+\sum_{i=1}^{k} \pi^{*}\left(a_{i}\right) \cdot \omega_{i},
$$

où $\alpha$ est une 1 -forme de $N, a_{i}$ des fonctions de $N$ et $\omega_{i}$ les 1 -formes verticales induites par une base orthonormée de $\mathcal{G}^{*}$. On a alors :

$$
\mathrm{d} \varphi=\pi^{*}\left(\mathrm{~d} \alpha+\sum_{i=1}^{k} a_{i} \cdot e_{i}\right)+\sum_{i=1}^{k} \mathrm{~d} \pi^{*}\left(a_{i}\right) \wedge \omega_{i},
$$

où $e_{i}$ désigne l'image de $\omega_{i}$ par l'application $e: \mathcal{G}^{*} \rightarrow \mathcal{H}^{2}(N)$. De plus, pour tout $i$ on a

$$
\begin{aligned}
\left\|\delta\left(\pi^{*}\left(a_{i}\right) \omega_{i}\right)\right\|^{2} & =\left(\delta\left(\pi^{*}\left(a_{i}\right) \omega_{i}\right), \delta\left(\pi^{*}\left(a_{i}\right) \omega_{i}\right)\right) \\
& =\left(\pi^{*}\left(a_{i}\right) \omega_{i}, \mathrm{~d} \delta\left(\pi^{*}\left(a_{i}\right) \omega_{i}\right)\right),
\end{aligned}
$$

où $(\cdot, \cdot)$ désigne le produit scalaire $L^{2}$. Comme $\pi^{*}\left(a_{i}\right) \omega_{i}$ est une forme $T^{k}$ invariante, $\delta\left(\pi^{*}\left(a_{i}\right) \omega_{i}\right)$ est une fonction invariante, c'est-à-dire que c'est le relevé d'une fonction sur $N$. Par conséquent, $\mathrm{d} \delta\left(\pi^{*}\left(a_{i}\right) \omega_{i}\right)$ est le relevé d'une 1 -forme sur $N$, et est donc orthogonale à $\pi^{*}\left(a_{i}\right) \omega_{i}$. Finalement, on a :

$$
\delta \varphi=\pi^{*}(\delta \alpha) .
$$

Si $\varphi$ est harmonique, on a $\mathrm{d} \varphi=0$ et $\delta \varphi=0$, donc

$$
\delta \alpha=0, \mathrm{~d} a_{i}=0 \text { pour tout } i, \text { et } \mathrm{d} \alpha+\sum_{i=1}^{k} a_{i} \cdot e_{i}=0 .
$$

Comme les fonctions $a_{i}$ sont constantes, $\sum_{i=1}^{k} a_{i} \cdot e_{i}$ est une 2 -forme harmonique de $N$, donc orthogonale à la forme exacte $\mathrm{d} \alpha$. On a donc $\Delta \alpha=0$ et $\sum_{i=1}^{k} a_{i}$. $e_{i}=0$. Comme $e$ est injective, les $e_{i}$ forment une famille libre, et donc $a_{i}=0$ pour tout $i$. On obtient finalement que les 1-formes harmoniques de $M$ sont les relevés des 1-formes harmoniques de $N$.

Pour majorer la première valeur propre non nulle du laplacien, il suffit de calculer le quotient de Rayleigh pour la métrique $g_{\varepsilon}$ d'une 1-forme orthogonale aux formes harmoniques. En notant $\left(\omega_{i}\right)$ les 1-formes verticales induites par la base duale de $\mathcal{B}_{\varepsilon}$, on choisit comme forme test $\varepsilon^{-1} \omega_{1}$. On vient de voir que la codifférentielle d'une telle forme est nulle, donc on peut écrire

$$
R\left(\varepsilon^{-1} \omega_{1}\right)=\frac{\left\|\mathrm{d}\left(\varepsilon^{-1} \omega_{1}\right)\right\|_{g_{\varepsilon}}^{2}}{\left\|\varepsilon^{-1} \omega_{1}\right\|_{g_{\varepsilon}}^{2}} .
$$

La forme $\varepsilon^{-1} \omega_{1}$ est indépendante de $\varepsilon$, donc $\mathrm{d}\left(\varepsilon^{-1} \omega_{1}\right)$ aussi, et comme de plus elle est horizontale donc sa norme ponctuelle ne dépend pas de $\varepsilon$; on a donc $\left\|\mathrm{d}\left(\varepsilon^{-1} \omega_{1}\right)\right\|^{2}=c \operatorname{Vol}\left(M, g_{\varepsilon}\right)$. Par ailleurs, $\left\|\varepsilon^{-1} \omega_{1}\right\|_{g_{\varepsilon}}^{2}=\varepsilon^{-1}\left\|\omega_{1}\right\|_{g_{\varepsilon}}^{2}$, donc 
$\left\|\varepsilon^{-1} \omega_{1}\right\|_{g_{\varepsilon}}^{2}$ est de la forme $c^{\prime} \varepsilon^{-2} \operatorname{Vol}\left(M, g_{\varepsilon}\right)$. Comme $\lambda_{1,1}\left(M, g_{\varepsilon}\right) \leq R\left(\varepsilon^{-1} \omega_{1}\right)$, on en déduit que $\lambda_{1,1}\left(M, g_{\varepsilon}\right) \leq C^{\prime} \cdot \varepsilon^{2}$, où $C^{\prime}$ est une constante dépendant des choix de $N, k$ et $e$.

Le cas $p=2$ se déduit du premier par la théorie de Hodge, en remarquant que dans le cas $p=1$ on a trouvé une petite valeur propre du laplacien retreint aux formes cofermées.

fait 5.6. - Si $b_{1}(N)>b_{2}(M)$, alors il existe une constante $C^{\prime \prime}(N, k, e)>0$ telle que pour tout $\varepsilon$, on a $\lambda_{p, b_{1}(N)-b_{2}(M)}\left(M, g_{\varepsilon}\right) \leq C^{\prime \prime} \cdot \varepsilon^{2}$ pour $p=2$ et 3 .

Démonstration. - On va se restreindre une nouvelle fois aux formes cofermées pour montrer le résultat pour $p=2$ et ensuite en déduire le cas $p=3$.

Contrairement au cas des 1-formes, on a pas en général de moyen simple de déterminer quelles sont les 2-formes harmoniques (on rencontre la même difficulté dans l'étude du spectre des fibrés en cercles, voir [CC00]). On va majorer le quotient de Rayleigh sur un espace test de dimension $b_{1}(N)$, et la condition $b_{1}(N)>b_{2}(M)$ assurera qu'on a bien majoré une ou plusieurs valeur propres non nulles.

En notant toujours $\left(\omega_{i}\right)$ les 1-formes verticales induites par la base duale de $\mathcal{B}_{\varepsilon}$ on considère une forme différentielle $\varphi=\omega_{1} \wedge \pi^{*}(\alpha)$, où $\alpha$ est une 1-forme harmonique de $N$. Son quotient de Rayleigh est

$$
R(\varphi)=\frac{\left\|\mathrm{d}\left(\omega_{1} \wedge \pi^{*}(\alpha)\right)\right\|_{g_{\varepsilon}}^{2}+\left\|\delta\left(\omega_{1} \wedge \pi^{*}(\alpha)\right)\right\|_{g_{\varepsilon}}^{2}}{\left\|\omega_{1} \wedge \pi^{*}(\alpha)\right\|_{g_{\varepsilon}}^{2}}
$$

La forme $\varphi$ est cofermée. En effet, si on calcule la différentielle de sa forme duale, on obtient :

$$
\begin{aligned}
\mathrm{d} *\left(\omega_{1} \wedge \pi^{*}(\alpha)\right) & =\mathrm{d}\left(\left(\bigwedge_{i \neq 1} \omega_{i}\right) \wedge \pi^{*}(* \alpha)\right) \\
& =\sum_{i \neq 1}(-1)^{i} \bigwedge_{j \neq 1, i} \omega_{j} \wedge \pi^{*}\left(e\left(\omega_{i}\right)\right) \wedge \pi^{*}(* \alpha)
\end{aligned}
$$

Comme $\pi^{*}\left(e\left(\omega_{i}\right)\right) \wedge \pi^{*}(* \alpha)=\pi^{*}\left(e\left(\omega_{i}\right) \wedge * \alpha\right)$ et que $e\left(\omega_{i}\right) \wedge * \alpha$ est de degré supérieur à la dimension de $N$, on en déduit que tous les termes de la somme sont nuls. On est ramené à :

$$
R(\varphi)=\frac{\left\|\mathrm{d}\left(\omega_{1} \wedge \pi^{*}(\alpha)\right)\right\|_{g_{\varepsilon}}^{2}}{\left\|\omega_{1} \wedge \pi^{*}(\alpha)\right\|_{g_{\varepsilon}}^{2}}
$$

On a d'une part $\left|\omega_{1} \wedge \pi^{*}(\alpha)\right|_{g_{\varepsilon}}^{2}=\left|\omega_{1}\right|_{g_{\varepsilon}}^{2}\left|\pi^{*}(\alpha)\right|_{g_{\varepsilon}}^{2}=|\alpha|^{2}$ et donc

$$
\left\|\omega_{1} \wedge \pi^{*}(\alpha)\right\|_{g_{\varepsilon}}^{2}=\|\alpha\|^{2}
$$

et d'autre part

$$
\left\|\mathrm{d}\left(\omega_{1} \wedge \pi^{*}(\alpha)\right)\right\|_{g_{\varepsilon}}^{2}=\left\|\mathrm{d} \omega_{1} \wedge \pi^{*}(\alpha)\right\|_{g_{\varepsilon}}^{2} \leq\left\|\mathrm{d} \omega_{1}\right\|_{g_{\varepsilon}}^{2}\|\alpha\|^{2} .
$$


On voit finalement que $R(\varphi) \leq R\left(\omega_{1}\right)$ quel que soit $\alpha$. La majoration de $R\left(\omega_{1}\right)$ obtenue précédemment permet de conclure.

On va maintenant donner des exemples de fibrés pour lesquels la condition $b_{1}(N)>b_{2}(M)$ est bien vérifiée.

Exemple 5.7. - On considère pour tout entier $k$ la variété $M=\left(S^{3}\right)^{k} \times S^{1}$. La fibration de Hopf $S^{1} \hookrightarrow S^{3} \rightarrow S^{2}$ induit une action libre de $T^{k}=\left(S^{1}\right)^{k}$ sur $\left(S^{3}\right)^{k}$, et permet donc de définir une fibration principale $T^{k} \hookrightarrow M \rightarrow$ $N$, où $N=\left(S^{2}\right)^{k} \times S^{1}$. La classe d'Euler $e$ de ce fibré est bien injective : l'image de $e$ est engendrée par les relevés à $M$ de chacune des classes d'Euler correspondant aux fibrations de Hopf qui sont bien linéairement indépendants. Enfin, la formule de Künneth permet de calculer aisément les nombres de Betti des produits $M$ et $N$, on obtient en particulier $b_{1}(N)=1$ et $b_{2}(M)=0$.

Remarque 5.8. - Dans l'exemple précédent, les métriques sur $M$ et $N$ ne sont a priori pas des métriques produits. On ne peut donc pas déduire directement les propriétés du spectre de la formule de Künneth.

Pour justifier la remarque 1.5 faite dans l'introduction, on va construire des contre-exemples aux théorèmes 1.1 et 1.2 de [CT97]. Ceux-ci permettent de montrer (cf. [Jam05]) que si, pour un réel $r$ tel que $0<r<\operatorname{inj}(M)$, on note $q$ le nombre de boules géodésiques de rayon $4^{-n} r$ nécessaire pour recouvrir $M$, alors il existe une constante $C$ ne dépendant que de la dimension et de la borne sur la courbure telle que

$$
\lambda_{p, 1}(M, g) \geq C r^{-2} q^{-7(p+1)} .
$$

C'est cette inégalité qu'on va contredire.

Exemple 5.9. - À courbure bornée et dimension $n$ fixée, le nombre $q$ de boules géodésiques de rayon $4^{-n} r$ nécessaire pour recouvrir $M$ est de l'ordre de $\operatorname{Vol}(M) \cdot r^{-n}$ quand $r$ est petit. Pour $p=1$, la minoration de l'inégalité 5.21 est donc de l'ordre de $\operatorname{Vol}(M)^{-14} r^{14 n-2}$.

On se donne un fibré $T^{k} \hookrightarrow M \rightarrow N$ et un effondrement $g_{\varepsilon}$ fournis par le théorème 1.3. On a alors $\operatorname{Vol}(M)=\varepsilon$ et on peut choisir $r \sim \varepsilon^{\frac{1}{k}}$, ce qui donne $q \sim \varepsilon^{1-n / k}$ et une minoration de l'ordre de $\varepsilon^{(14 n-14 k-2) / k}=\varepsilon^{(14 d-2) / k}$ en notant $d=n-k$ la dimension de la base $N$.

Or, si on fixe la dimension $d \geq 3$ de $N$ et qu'on choisit $k$ suffisamment grand (c'est possible puisque $b_{2}(N)$ peut être arbitrairement grand à dimension fixée), l'estimation $\varepsilon^{(14 d-2) / k}$ ne peut pas minorer $\lambda_{1,1}\left(M, g_{\varepsilon}\right)$ qui est de l'ordre de $\varepsilon^{2}$ quand $\varepsilon$ tend vers 0 .

Il reste enfin à démontrer le corollaire 1.7. 
Démonstration. - Cas $n=5$ : On considère une variété compacte $N$ de dimension 3 telle que $b_{2}(N) \geq 2$ et on choisit pour $M$ un fibré principal en tore $T^{2}$ sur $N$ dont la classe d'Euler est injective. Les résultats précédents assure l'existence d'un effondrement tel que $\lambda_{p, 1}(M) \leq C \cdot \operatorname{inj}(M)^{4}$ pour $p$ égal à 1 et 2. Par dualité de Hodge, on a la même inégalité pour $n-1$ et $n-2$, donc pour tout $p$ entre 1 et $n-1$.

Cas $n=7$ : On peut vérifier que le fibré construit dans l'exemple 5.7 pour $k=2$ convient. On sait déjà que $\lambda_{p, 1}(M) \leq C \cdot \operatorname{inj}(M)^{4}$ pour $p=1,2$ et 3 , et la dualité de Hodge donne l'inégalité pour $p=4,5$ et 6 .

\section{Minoration du spectre des fibrés principaux en tores}

6.1. Minoration de la première valeur propre des 1-formes. - Les résultats des sections 2 à 4 nous permettent de démontrer le théorème 1.8 . On a vu qu'on pouvait se ramener au cas d'un fibré muni d'une métrique adaptée. On va donc montrer le résultat du théorème 1.8 pour un fibré vérifiant les conclusions du théorème 3.1 :

Théorème 6.1. - Soit $a>0, d>0$ deux réels, $n, k$ et $m$ trois entiers tels que $n=k+m$, et $\left(N^{m}, h\right)$ une variété riemannienne. Il existe des constantes $c(n, a, d,(N, h))$ et $\varepsilon(n, a, d,(N, h))$ strictement positives telles que si $\bar{g}$ est une métrique sur le tore $T^{k}$ telle que $\operatorname{diam}\left(T^{k}\right)<\varepsilon$ et si $T^{k} \hookrightarrow M^{n} \rightarrow N$ est un fibré principal muni d'un couple de métriques $(g, h)$ adapté au fibré et tel que $g=\bar{g}$ en restriction à la fibre, $\operatorname{diam}(M, g)<d$ et $\left|K_{M}(X, Y)\right| \leq$ a pour toute paire $(X, Y)$ de vecteurs horizontaux orthonormés, alors on a

$$
\lambda_{1,1}(M, g) \geq c \cdot \operatorname{Vol}^{2}\left(T^{k}\right) .
$$

On s'est ici donné comme hypothèse que la métrique sur $N$ est fixée. En effet, une hypothèse sur la courbure ne nous sera pas suffisante. Par ailleurs la minoration du spectre en fonction du rayon d'injectivité découle du fait que le rayon d'injectivité de $M$ est égal à celui de la fibre si $\varepsilon$ est suffisamment petit (la fibre étant totalement géodésique) et que, la restriction de la métrique à la fibre étant plate, on a $\operatorname{Vol}\left(T^{k}\right) \geq C \operatorname{inj}\left(T^{k}\right)^{k}$ où $C$ ne dépend que de $k$.

Dans un premier temps, nous allons démontrer le théorème dans le cas où le fibré $M$ ne contient pas de sous-fibré trivial, c'est-à-dire quand l'application $e: \mathcal{G}^{*} \rightarrow \mathcal{H}^{2}(N, h)$ est injective. Nous généraliserons ensuite le résultat à un fibré principal quelconque. D'autre part, on se restreindra aux formes $T^{k}$ invariantes, en vertu des résultats de la section 4 (corollaire 4.1). En effet, le spectre des formes orthogonales aux formes invariantes sera minoré en fonction de la constante $\varepsilon$ du théorème, et on pourra toujours choisir cette constante suffisamment petite de sorte que le spectre des formes orthogonales aux formes invariantes soit plus grand que le terme $c \cdot \operatorname{Vol}^{2}\left(T^{k}\right)$. 
Supposons donc $e$ injective. La démonstration se déroule en deux étapes. D'abord, on se ramène à l'étude des valeurs propres de l'opérateur $e^{*} e$, l'adjoint étant défini en munissant $\mathcal{H}^{2}(N)$ de sa norme $L^{2}$ :

fait 6.2. - Il existe $\varepsilon\left(n, a, \lambda_{0,1}(N, h), \lambda_{1,1}(N, h)\right)>0$ et $c\left(n, a, \lambda_{0,1}(N, h)\right)>$ 0 tels que pour toute 1-forme $\varphi$ sur $M T^{k}$-invariante et orthogonale $\grave{a}$ $\operatorname{Ker} \Delta^{1}(M, g)$, si le quotient de Rayleigh de $\varphi$ vérifie $R(\varphi)<\varepsilon$, alors il existe une forme $\omega$ induite par un élément de $\mathcal{G}^{*}$ telle que $\|e(\omega)\|^{2} \leq c \cdot \varepsilon\|\omega\|^{2}$.

Démonstration. - Soit $\varphi$ une 1-forme différentielle $T^{k}$-invariante de $M$. On peut comme dans la section précédente écrire $\varphi$ sous la forme

$$
\varphi=\pi^{*}(\alpha)+\sum_{i=1}^{k} \pi^{*}\left(a_{i}\right) \cdot \omega_{i},
$$

où $\alpha$ est une 1-forme de $N, a_{i}$ des fonctions de $N$ et $\omega_{i}$ les 1 -formes verticales induites par une base orthonormée de $\mathcal{G}^{*}$. On a alors :

$$
\mathrm{d} \varphi=\pi^{*}\left(\mathrm{~d} \alpha+\sum_{i=1}^{k} a_{i} \cdot e_{i}\right)+\sum_{i=1}^{k} \mathrm{~d} \pi^{*}\left(a_{i}\right) \wedge \omega_{i},
$$

où $e_{i}$ désigne l'image de $\omega_{i}$ par l'application $e: \mathcal{G}^{*} \rightarrow \mathcal{H}^{2}(N)$. On a vu de plus dans la démonstration du fait 5.5 que $\varphi$ vérifie

$$
\delta \varphi=\pi^{*}(\delta \alpha),
$$

et que le fait que $e$ soit injectif implique que les 1-formes harmoniques de $M$ sont exactement les relevés des 1-formes harmoniques de $N$.

Supposons que $\varphi$ est de norme 1 , c'est-à-dire que $\|\alpha\|^{2}+\sum_{i=1}^{k}\left\|a_{i}\right\|^{2}=1$, et qu'elle est orthogonale aux formes harmoniques de $M$. Le quotient de Rayleigh de $\varphi$ s'écrit alors

$$
R(\varphi)=\|\delta \alpha\|^{2}+\left\|\mathrm{d} \alpha+\sum_{i=1}^{k} a_{i} \cdot e_{i}\right\|^{2}+\sum_{i=1}^{k}\left\|\mathrm{~d} \pi^{*}\left(a_{i}\right)\right\|^{2} .
$$

Supposons que $R(\varphi)<\varepsilon$ pour un $\varepsilon>0$ donné. On a en particulier $\| \mathrm{d} \alpha+$ $\sum_{i=1}^{k} a_{i} \cdot e_{i} \|^{2}<\varepsilon$. Pour tout $i$, notons $\bar{a}_{i}$ la valeur moyenne de la fonction $a_{i}$. On peut alors écrire

$$
\left\|\mathrm{d} \alpha+\sum_{i=1}^{k} \bar{a}_{i} \cdot e_{i}+\sum_{i=1}^{k}\left(a_{i}-\bar{a}_{i}\right) \cdot e_{i}\right\|^{2}<\varepsilon .
$$

L'inégalité triangulaire nous donne alors :

$$
\left\|\mathrm{d} \alpha+\sum_{i=1}^{k} \bar{a}_{i} \cdot e_{i}\right\|<\left\|\sum_{i=1}^{k}\left(a_{i}-\bar{a}_{i}\right) \cdot e_{i}\right\|+\sqrt{\varepsilon} .
$$


Comme les fonctions $\left(a_{i}-\bar{a}_{i}\right)$ sont de moyennes nulles, leur quotient de Rayleigh est supérieur à $\lambda_{0,1}(N, h)$, et donc $\left\|a_{i}-\bar{a}_{i}\right\|^{2} \leq \frac{\left\|\mathrm{d} a_{i}\right\|^{2}}{\lambda_{0,1}(N, h)} \leq \frac{\varepsilon}{\lambda_{0,1}(N, h)}$. Par ailleurs, la forme exacte $\mathrm{d} \alpha$ est orthogonale à la forme harmonique $\sum_{i=1}^{k} \bar{a}_{i} \cdot e_{i}$. On a donc finalement

$$
\begin{aligned}
\left\|\sum_{i=1}^{k} \bar{a}_{i} \cdot e_{i}\right\|^{2} & \leq\left\|\mathrm{d} \alpha+\sum_{i=1}^{k} \bar{a}_{i} \cdot e_{i}\right\|^{2} \\
& \leq\left(\left\|\sum_{i=1}^{k}\left(a_{i}-\bar{a}_{i}\right) \cdot e_{i}\right\|+\sqrt{\varepsilon}\right)^{2} \\
& \leq\left(\sum_{i=1}^{k}\left(\left\|a_{i}-\bar{a}_{i}\right\| \cdot\left\|e_{i}\right\|_{\infty}\right)+\sqrt{\varepsilon}\right)^{2} \\
& \leq \varepsilon\left(1+\frac{\sum_{i=1}^{k}\left\|e_{i}\right\|_{\infty}}{\sqrt{\lambda_{0,1}(N, h)}}\right)^{2} .
\end{aligned}
$$

Le lemme 3.2 permet d'obtenir une majoration de $\left\|\sum_{i=1}^{k} \bar{a}_{i} \cdot e_{i}\right\|^{2}$ en fonction de $n, \lambda_{0,1}(N, h)$ et d'une borne sur la courbure de $(M, g)$.

Pour tout $i$, la fonction $a_{i}-\bar{a}_{i}$ est orthogonale à $\bar{a}_{i}$, donc

$$
\|\alpha\|^{2}+\sum_{i=1}^{k}\left(\left\|\bar{a}_{i}\right\|^{2}+\left\|a_{i}-\bar{a}_{i}\right\|^{2}\right)=1 .
$$

D'une part, on a déjà vu que chaque terme $\left\|a_{i}-\bar{a}_{i}\right\|^{2}$ est majoré par $\frac{\varepsilon}{\lambda_{0,1}(N, h)}$. D'autre part, comme $\varphi$ est orthogonale aux relevés des formes harmoniques de $(N, h), \alpha$ est elle-même orthogonale aux formes harmoniques de $N$. On peut donc écrire

$$
\|\alpha\|^{2} \leq \frac{1}{\lambda_{1,1}(N, h)}\left(\|\mathrm{d} \alpha\|^{2}+\|\delta \alpha\|^{2}\right) .
$$

Le terme $\|\delta \alpha\|^{2}$ est majoré par $R(\varphi)$, donc par $\varepsilon$, et $\|\mathrm{d} \alpha\|^{2}$ est majoré en fonction de $a, n$ et $\lambda_{0,1}(N, h)$ comme dans l'inégalité 6.7. Il découle donc de l'équation (6.8):

$$
\sum_{i=1}^{k}\left\|\bar{a}_{i}\right\|^{2} \geq 1-\tau\left(n, a, \lambda_{0,1}(N, h), \lambda_{1,1}(N, h)\right) \varepsilon .
$$

Si on prend pour $\omega$ la 1 -forme $\sum_{i=1}^{k} \bar{a}_{i} \omega_{i}$, on a

$$
\frac{\|e(\omega)\|^{2}}{\|\omega\|^{2}}=\frac{\left\|\sum_{i=1}^{k} \bar{a}_{i} \cdot e_{i}\right\|^{2}}{\sum_{i=1}^{k}\left\|\bar{a}_{i}\right\|^{2}}
$$


Selon 6.10 , le dénominateur est supérieur à $\frac{1}{2}$ si $\varepsilon$ est suffisamment petit. La majoration du numérateur fournie par (6.7) donne alors le résultat souhaité.

On va maintenant minorer le spectre de $e^{*} e$ en fonction du volume de la fibre $T^{k}$.

fait 6.3. - Il existe une constante $c(n, a,(N, h))>0$ telle que la première valeur propre de e e soit minorée par $c \cdot \operatorname{Vol}\left(T^{k}\right)^{2}$.

Démonstration. - Soient $\lambda_{1}, \cdots, \lambda_{k}$ les valeurs propres de $e^{*} e$ classées dans l'ordre croissant. Comme $e$ est injective, ces valeurs propres sont non nulles et la première vérifie

$$
\lambda_{1}=\frac{\prod_{i} \lambda_{i}}{\prod_{i \neq 1} \lambda_{i}}=\frac{\operatorname{Det}\left(e^{*} e\right)}{\prod_{i \neq 1} \lambda_{i}} .
$$

Par ailleurs, les valeurs propres de $e^{*} e$ vérifient $\lambda_{i} \leq\left\|e^{*} e\right\|$, donc

$$
\lambda_{1} \geq \frac{\operatorname{Det}\left(e^{*} e\right)}{\left\|e^{*} e\right\|^{k-1}} \geq \frac{\operatorname{Det}\left(e^{*} e\right)}{\|e\|^{2 k-2}} .
$$

L'image de $e$ est un sous-espace de $\operatorname{Ker} \Delta^{2}(N)$ de dimension $k$, engendré par un sous-réseau du réseau des formes harmoniques entières de $N$. Si on restreint $e$ et $e^{*}$ à ce sous-espace, on peut écrire $\operatorname{Det}\left(e^{*} e\right)=(\operatorname{Det} e)^{2}$, où Det $e$ est le déterminant d'une matrice de $e$ écrite dans des bases orthonormées de $\mathcal{G}^{*}$ et $\operatorname{Im} e$, ce qui donne

$$
\lambda_{1} \geq \frac{(\text { Det } e)^{2}}{\|e\|^{2 k-2}} .
$$

Le lemme 3.2 donne une majoration de $\|e\|$ en fonction de $n$ et de la borne $a$ sur la courbure de $M$, il ne reste donc qu'à minorer Det $e$. Notons $\operatorname{Det}^{\prime} e$ le déterminant de la matrice de $e$ dans la base canonique de $\mathcal{G}^{*}=\mathbb{R}^{k^{*}}$ et une base orthonormée de $\operatorname{Im} e$. On a alors Det $e=\left(\operatorname{Det}^{\prime} e\right)\left(\operatorname{Vol} T^{k}\right)$. Comme les images dans $\operatorname{Ker} \Delta^{2}(N)$ des éléments de la base canonique de $\mathcal{G}^{*}$ sont des formes entières, le déterminant $\operatorname{Det}^{\prime} e$, qui est aussi le volume de $e\left([0,1]^{k}\right)$, est un multiple du volume d'un domaine fondamental du réseau des formes entières dans $\operatorname{Im} e$. Comme par ailleurs $\operatorname{Det}^{\prime} e$ est non nul, il sera donc minoré par le volume de ce domaine fondamental. Si on note $\rho$ le minimum des normes des 2-formes harmoniques entières non nulles, ce volume est minoré par le volume d'une boule de rayon $\frac{\rho}{2}$ dans $\operatorname{Im} e$, et donc minoré par une constante ne dépendant que de $n$ et de la métrique $h$ de $N$. On peut donc bien écrire

$$
\lambda_{1} \geq c(n, a,(N, h)) \cdot \operatorname{Vol}\left(T^{k}\right)^{2} .
$$


Nous allons maintenant supposer que $e$ n'est pas injective. Notons $l$ la dimension de son noyau. Le premier nombre de Betti de $M$ est alors $b_{1}(N)+l$. En effet, on a vu que si une 1-forme $\varphi=\pi^{*}(\alpha)+\sum_{i=1}^{k} \pi^{*}\left(a_{i}\right) \cdot \omega_{i}$ est harmonique, cela signifie, d'après 6.2 et 6.3 :

$$
\Delta \alpha=0, \mathrm{~d} a_{i}=0 \text { pour tout } i, \text { et } \sum_{i=1}^{k} a_{i} \cdot e_{i}=0 .
$$

Comme les fonctions $a_{i}$ sont constantes, l'ensemble des $a_{i}$ tels que $\sum_{i=1}^{k} a_{i} \cdot e_{i}=$ 0 est exactement le noyau de $e$. L'espace des formes harmoniques de $M$ est donc l'espace engendré par les relevés des formes harmoniques de $N$ et les formes verticales induites par les éléments de noyau de $e$.

On peut reprendre la démonstration précédente en prenant pour $\left(\omega_{i}\right)_{i}$ une base de $\mathcal{G}^{*}$ telle que $\omega_{k-l+1}, \cdots, \omega_{k}$ soit une base de Ker $e$ (le fait que la forme $\varphi$ est orthogonale aux formes harmoniques se traduit par le fait que $a_{k-l+1}, \cdots, a_{k}=0$ ) et en étudiant $e^{*} e$ restreint à l'orthogonal de Ker $e$. On obtient de la même façon le résultat du fait 6.2 , à savoir que la première valeur propre du laplacien sur $M$ est minorée à une constante multiplicative près par la première valeur propre de $\left(e^{*} e\right)_{\mid(\operatorname{Ker} e)^{\perp}}$.

Pour minorer le spectre de $\left(e^{*} e\right)_{\mid(\operatorname{Ker} e)^{\perp}}$, on doit être un peu plus attentif dans la manipulation des bases de $\mathcal{G}^{*}$.

Soit $\mathcal{B}=\left(\omega_{1}, \cdots, \omega_{k}\right)$ une base orthonormée de $\mathcal{G}^{*}$ et $\mathcal{B}^{\prime}=\left(\omega_{1}^{\prime}, \cdots, \omega_{k}^{\prime}\right)$ une base du réseau des entiers de $\mathcal{G}^{*}$, telles que $\left(\omega_{1}, \cdots, \omega_{l}\right)$ et $\left(\omega_{1}^{\prime}, \cdots, \omega_{l}^{\prime}\right)$ soient des bases de Ker $e$ (comme l'image du réseau des entiers de $\mathcal{G}^{*}$ est contenue dans un réseau, le noyau de $e$ est effectivement engendré par des éléments entiers). La matrice de passage de $\mathcal{B}$ à $\mathcal{B}^{\prime}$ est de la forme

$$
P=\left(\begin{array}{cc}
P_{1} & P_{2} \\
0 & P_{3}
\end{array}\right)
$$

où $P_{1}$ est un bloc carré de taille $l$. Si se donne une base orthonormée de $\operatorname{Im} e$, la matrice de $e$ s'écrit sous la forme $(0, A)$ dans la base $\mathcal{B}$ et $\left(0, A^{\prime}\right)$ dans la base $\mathcal{B}^{\prime}$, où $A$ et $A^{\prime}$ sont des blocs carrés de taille $k-l$ et vérifient $A^{\prime}=A P_{3}$.

Le spectre de $\left(e^{*} e\right)_{\mid(\text {Ker } e)^{\perp}}$ est celui de $A^{*} A$. On peut écrire, comme dans la démonstration du fait 6.3 :

$$
\lambda_{1} \geq \frac{\operatorname{Det} A^{*} A}{\left\|A^{*} A\right\|^{k-l-1}} \geq \frac{(\operatorname{Det} A)^{2}}{\|A\|^{2(k-l-1)}},
$$

où $\lambda_{1}$ est la première valeur propre non nulle de $e^{*} e$. De plus, on a Det $A^{\prime}=$ Det $A \cdot \operatorname{Det} P_{3}$ et donc

$$
\operatorname{Det} A=\frac{\operatorname{Det} A^{\prime}}{\operatorname{Det} P_{3}}=\operatorname{Det} A^{\prime} \frac{\operatorname{Det} P_{1}}{\operatorname{Det} P} .
$$


Le déterminant de $A^{\prime}$ est, comme précédemment, minoré par le covolume du réseau des formes entières dans $\operatorname{Im} e$, et $\operatorname{Det} P$ s'interprète géométriquement comme l'inverse du volume de $T^{k}$.

Il reste à minorer Det $P_{1}$. Comme Ker $e$ est engendré par des éléments entiers de $\mathcal{G}^{*}$, l'orthogonal de Ker $e$ pour la dualité définit un sous-tore $T^{k-l}$ de $T^{k}$. De plus, le dual de de l'algèbre de Lie $\mathcal{G}\left(T^{k} / T^{k-l}\right)$ du quotient $T^{k} / T^{k-l}$ est isomorphe à Ker $e$. La matrice $P_{1}$ est donc la matrice de passage d'une base orthonormée de $\mathcal{G}^{*}\left(T^{k} / T^{k-l}\right)$ dans une base du réseau des entiers de $\mathcal{G}^{*}\left(T^{k} / T^{k-l}\right)$, et par conséquent Det $P_{1}$ est l'inverse du volume de $T^{k} / T^{k-l}$ pour la métrique quotient. Le diamètre de $T^{k} / T^{k-l}$ est majoré par $\varepsilon$, comme celui de $T^{k}$, et par conséquent son volume aussi.

\section{Références}

[AlK00] J. A. Álvarez López \& Y. A. Kordyukov - «Adiabatic limits and spectral sequences for riemannian foliations », Geom. funct. anal. 10 (2000), no. 5, p. $977-1027$.

[Bes08] A. L. Besse - Einstein Manifolds, Classics in Mathematics, Springer Verlag, 2008.

[BT82] R. Вотт \& L. W. Tu - Differential form in algebraic topology, Springer Verlag, 1982.

[Car84] Y. CARRIÈRE - «Les propriétés topologiques des flots riemanniens retrouvées à l'aide du théorème des variétés presque plates », Math. Z. 186 (1984), p. 393-400. [CC90] B. Colbois \& G. Courtois - « A note on the first non zero eigenvalue of the Laplacian acting on $p$-forms », Manuscripta Math. 68 (1990), no. 2, p. 143-160. [CC00] _ « Petites valeurs propres des $p$-formes différentielles et classe d'Euler des $S^{1}$-fibrés », Ann. scient. Éc. norm. sup. (4) 33 (2000), no. 5, p. 611-645.

[CFG92] J. Cheeger, K. Fukaya \& M. Gromov - « Nilpotent structures and invariant metrics on collapsed manifolds », J. Amer. Math. Soc. 5 (1992), no. 2, p. $327-372$.

[CG86] J. Cheeger \& M. Gromov - « Collapsing riemannian manifolds while keeping their curvature bounded I», J. Differential Geom. 23 (1986), no. 3, p. 309-346. [CT97] S. Chanillo \& F. TrÈves - « On the lowest eigenvalue of the Hodge Laplacian », J. Differ. Geom. 45 (1997), no. 2, p. 273-287.

[Dod82] J. Dodziuk - «Eigenvalues of the Laplacian on forms », Proc. of Am. Math. Soc. 85 (1982), no. 3, p. 438-443.

[For95] R. Forman - « Spectral Sequences and Adiabatic Limits», Comm. Math. Phys. 168 (1995), no. 1, p. 57-116.

[GHL87] S. Gallot, D. Hulin \& J. Lafontaine - Riemannian Geometry, Springer Verlag, 1987.

[Gro80] M. Gromov - « Paul Levy's isoperimetric inegality », Prépublication IHÉS (1980), paru dans Gro99. 
[Gro99] _ Metric Structures for Riemannian and Non-Riemannian Spaces, Birkhäuser, 1999.

[Her60] R. Hermann - «A sufficient condition that a mapping of Riemannian manifold be a fibre bundle », Proc. of Am. Math. Soc. 11 (1960), p. 236-242.

[Jam03] P. JAmmes - «Sur le spectre des fibrés en tore qui s'effondrent », Manuscripta Math. 110 (2003), no. 1, p. 13-31.

[Jam05] _ _ «Effondrements et petites valeurs propres des formes différentielles », Sémin. théor. spectr. géom. 23 (2005), p. 115-124.

[Jam10] _ _ « Effondrement, spectre et propriétés diophantiennes des flots riemanniens », Ann. inst. Fourier 60 (2010), no. 1, p. 257-290.

[Jam11] _ _ « Autour de la géométrie du laplacien de Hodge-de Rham », Habilitation à diriger des recherches, université Nice Sophia Antipolis, déc. 2011.

[Li80] P. LI - « On the Sobolev constant and the $p$-spectrum of a compact riemannian manifold », Ann. scient. Éc. norm. sup. (4) 13 (1980), p. 451-469.

[Lot02] J. LotT - «Collapsing and the differential form Laplacian : the case of a smooth limit space », Duke Math. Journal 114 (2002), no. 2, p. 267-306.

[LY80] P. Li \& S. YAU - « Estimates of eigenvalues of a compact riemannian manifold », in Proceedings Symposium on Pure Math., vol. 36, 1980, p. 205-239.

[Man08] T. Mantuano - «Discretization of Riemannian manifolds applied to the Hodge Laplacian », Amer. J. Math. 130 (2008), no. 6, p. 1477-1508.

[Ram05] S. RAmAnan - Global calculus, Graduate Studies in Mathematics, vol. 65, Amer. Math. Soc., 2005.

[Sch80] W. SCHMIDT - Diophatine approximations, Lecture notes in mathematics, vol. 785, Springer Verlag, 1980.

Pierre Jammes, Université Côte d'Azur, CNRS, LJAD, France

E-mail : pjammes@unice.fr 